\title{
Design of Feedback Controls Supporting TCP Based on the State-Space Approach
}

\author{
Ki Baek Kim
}

\begin{abstract}
This paper investigates how to design feedback controls supporting transmission control protocol (TCP) based on the state-space approach for the linearized system of the well-known additive increase multiplicative decrease (AIMD) dynamic model. We formulate the feedback control design problem as state-space models without assuming its structure in advance. Thereby, we get three results that have not been observed by previous studies on the congestion control problem.

1) In order to fully support TCP, we need a proportional-derivative (PD)-type state-feedback control structure in terms of queue length (or RTT: round trip time). This backs up the conjecture in the networking literature that the AQM RED is not enough to control TCP dynamic behavior, where RED can be classified as a P-type AQM (or as an output feedback control for the linearized AIMD model).

2) In order to fully support TCP in the presence of delays, we derive delay-dependent feedback control structures to compensate for delays explicitly under the assumption that RTT, capacity and number of sources are known, where all existing AQMs including RED, REM/PI and AVQ are delay-independent controls.

3) In an attempt to interpret different $A Q M$ structures in a unified manner rather than to compare them via simulations, we propose a PID-type mathematical framework using integral control action.

As a performance index to measure the deviation of the closedloop system from an equilibrium point, we use a linear quadratic (LQ) cost of the transients of state and control variables such as queue length, aggregate rate, jitter in the aggregate rate, and congestion measure. Stabilizing gains of the feedback control structures are obtained minimizing the LQ cost. Then, we discuss the impact of the control structure on performance using the PID-type mathematical framework. All results are extended to the case of multiple links and heterogeneous delays.
\end{abstract}

Index Terms-Active queue management, additive increase multiplicative decrease (AIMD), explicit delay compensation, optimal control, state-space approach, transmission control protocol (TCP).

\section{INTRODUCTION}

$\mathbf{O}$ $\mathrm{N}$ THE Internet, congestion control enables end-users to fully utilize the allocated capacity with the help of queueing at routers. Since end-users do not know the allocated capacity, a dynamic window-based mechanism transmission

Manuscript received January 8, 2004; revised May 27, 2005. Recommended by Associate Editor Y. Wardi. This work was funded in part by the AlcatelINRIA OSC "End-to-End Performance Evaluation of Packet Networks". This work is based on its companion technical reports and conference papers [1]-[5] (mainly on [1]).

The author is with Telecommunication Network, Samsung Electronics Co., Ltd., Suwon 442-600, Korea (e-mail: kibaek.kim@samsung.com).

Digital Object Identifier 10.1109/TAC.2006.878749 control protocol (TCP) acting at a packet level has been developed, so that each flow from an end-user obeys a "conservation of packets" principle for a connection "in equilibrium." Since congestion information occurs at routers, interaction between TCP and the congestion information cannot avoid delays which make the closed-loop dynamics difficult to deal with. Signaling the congestion information by dropping or marking packets at routers is called active queue management (AQM). Since TCP Reno/AQM Droptail has been proposed in [6], the current internet is still using this protocol and its variants as a congestion control strategy.

Droptail can cause a large variation of queueing delay since it drops packets when the queue is full. More importantly, Droptail can often cause the global synchronization [7]-[9] and thus have low throughput. In order to overcome these problems, random early detection (RED) has been suggested in [10], [11] whose drops probability is proportional to the average queue length. Since then, there have been a lot of investigations about how to tune the design parameters in RED [12]-[14]. The experimental results show that RED is not enough to control TCP and thus not easy to fully utilize the given network resources. As a result, new AQM algorithms such as adaptive virtual queue (AVQ) [15] and random exponential marking (REM) [16] have been suggested. However, none of these papers appropriately address how to tune the gains of their AQM structures for closed-loop stability since they lack of a TCP dynamic model. So, their AQM algorithms are only compared through simulations in the literature.

In order to address this problem, paper [17] has developed a dynamic model to reflect AIMD (Additive Increase Multiplicative Decrease) mode of TCP for a single link and homogeneous sources and paper [18] has applied the transfer function approach to the problem of stabilizing RED design based on the AIMD model. As a follow up, papers [19] and [20] have investigated how to scale gains of proportional-integral (PI)-type REM in terms of queue length and P-type AVQ in terms of aggregate, respectively. Papers [21] and [22] have suggested PI-type AQM in terms of aggregate in an inner loop and P-type AQM with a low-pass filter in terms of aggregate, respectively. Although all these papers suggest stabilizing conditions from the linearized systems, they have not focused on what kind of control structures are necessary to fully control the closed-loop system TCP/AQM fair.

In addition, none of those papers consider how to compensate for delays explicitly even if they know the previous dynamic information and delays. This kind of control strategy is called delay-independent (or memoryless) control in the literature. Along this line, the initial works in the Internet literature are [23] and [24] based on the optimization framework [25]. It 
is well-known that the delay-independent control has a limit on performance in the presence of a large delay [26] compared with the delay-dependent (or memory) control since the delay-independent controls cannot regulate the delayed closed-loop dynamics arbitrarily. In the literature, previous studies on feedback control design in the presence of delays have focused on deriving stabilizing conditions, while our work studies an explicit feedback control design.

This paper tries to address these issues as follows: In order to get basic results for more realistic cases, we study a simplified version of this problem as in [18], [19], and [21]-[24]. First, we consider the linearized system of the AIMD and queue dynamics around the equilibrium. So, stability means local stability near equilibrium and the variables denote transients from their equilibrium points. The study on the linearized system can be justified by the fact that congestion occurs near the equilibrium point since the main role of feedback control is to keep the closed-loop system around the equilibrium. Second, we assume that information of the networks is known when we try to compensate for delays and to get stabilizing optimal gains for the derived feedback control structure. In addition, we do not study the case of input/state constrained uncertain systems which are intrinsically included in real networks and network simulators. Instead, we study the case of multiple links and heterogeneous delays which to our knowledge, has never been investigated in the TCP Reno literature before our work [2].

The main new feature of the present paper is to formulate the feedback control design problem as state-space models. ${ }^{1}$ It allows us to investigate what is a natural state-feedback control, how to compensate for delays explicitly and what is the impact of different feedback control structures on performance.

In Section II, we introduce the well-known TCP AIMD model in [17], [18] and its extension to multiple links and heterogeneous sources.

In Section III, we derive the state-space model of the linearized AIMD and queue dynamics for the feedback control design. Thereby, we obtain the PD-type state-feedback control structure in terms of queue length (or RTT: round trip time) which implies the structural deficiency of P-type RED. We show that this procedure can easily be extended to the case of additional dynamics like the low-pass filter of RED. By applying integral control action, we propose a mathematical framework to include PI-type REM and PI as well as P-type REM, from which we study the impact of each structure on performance in Section V.

In Section IV, we suggest a delay-dependent feedback control to compensate for the delay in the congestion measure explicitly under the assumption that the forward delay from source to router is zero. This assumption is relaxed by adding a modified virtual queue so that we can still compensate for delays explicitly in the presence of both forward and backward delays. As a subsidiary result of this study, we verify that a simplified AVQ, which is P-type AQM in terms of aggregate, is a state-feedback control for the AIMD model based on the virtual queue dynamics.

\footnotetext{
${ }^{1}$ Since the state-space approach was developed in 1950s, it has been widely investigated in the literature due to many advantages over the transfer function approach (refer to any control literature for more details [27]).
}

In Section V, we obtain stabilizing gains of the control structures by minimizing a linear quadratic (LQ) cost of the transients of state and control variables. Thus, the optimal control framework enables us to measure deviation of transients from the equilibrium point in the form of quadratic cost which can be considered as a control Lyapunov function. For example, a slower transient will incur a higher cost. Inverse optimal control and the design of stabilizing gains by eigenvalues are also studied. Then, we discuss the impact of each structure on performance using the results of this study rather than to compare their performances via simulations. As a by-product of this study, we show that it is possible to obtain stabilizing gains with one design parameter by setting all eigenvalues of the closed-loop system to be the same.

In Section VI, all results are extended to the case of multiple links and heterogeneous sources. This study shows that the design procedures and resulting control structures for single link and homogeneous sources hold for general networks.

\section{Preliminary: The Well-Known AIMD Model AND SCOPE OF OUR STUDIES}

In this section, we introduce the well-known AIMD model of TCP in [17], [18] and its extension to multiple links and heterogeneous sources, and discuss our scope of this study.

\section{A. Notation}

For describing the well-known AIMD model, we will use the following notations.

- $N$ is the number of TCP sources, which we assume to be constant with time.

- $c_{l}$ is the capacity of link $l$ in packets/s.

- $d_{s}$ is the round trip propagation delay of source $s$.

- $b_{l}(t)$ is the real queue length of link $l$ at time $t$ (the average queue and virtual queue lengths are denoted as $\bar{b}_{l}(t)$ and $\tilde{b}_{l}(t)$, respectively).

- $\tau_{l_{s}}^{f}(t)$ is the forward delay from source $s$ to link $l$ at time $t$.

- $\tau_{l s}^{b}(t)$ is the backward delay from link $l$ to source $s$ at time $t$.

- $R_{l s}$ is a $\{0,1\}$-valued variable with value 1 if source $s$ uses link $l, 0$ otherwise.

- $\tau_{s}(t)$ is the RTT of source $s$ at time $t\left(\tau_{s}(t)=d_{s}+\right.$ $\left.\sum_{l} R_{l s}\left(b_{l}(t) / c_{l}\right)\right)$.

- $w_{s}(t)$ is the window size of source $s$ at time $t$.

- $p_{l}(t)$ is the feedback control at time $t$ for TCP (it is mainly assumed to be the loss probability in this paper, but it can have other quantity depending on what kind of congestion information is used for the feedback control).

- $y_{l}(t)$ is the aggregate of link $l$ at time $t$.

Equilibrium of each variable will be denoted as $b_{l}^{*}, \tau_{l s}^{f *}, \tau_{l s}^{b *}$, $\tau_{s}^{*}, w_{s}^{*}$ and $p_{l}^{*}$.

Whenever RTT, or forward and backward delay, appear in the argument of a variable, we will replace it by its equilibrium value $\tau_{s}^{*}, \tau_{l s}^{f *}$, and $\tau_{l s}^{b *}$. However, when round-trip time appears in the dependent variable, we will consider it time-varying. This avoids recursive time-arguments, but is admittedly an approximation, done exclusively for model tractability. Using this rule, $y_{l}(t)$ is approximated by $y_{l}(t) \approx \sum_{s} R_{l s}\left(w_{s}\left(t-\tau_{l s}^{f *}\right) / \tau_{s}(t-\right.$ $\left.\tau_{l s}^{f *}\right)$ ) (the rationale for the approximation is that the source rate, 
which is defined by $x_{s}(\cdot)$, and the window size are linked by a Little like law: $\left.x_{s}(t) \approx w_{s}(t) / \tau_{s}(t)\right)$.

In order to represent the linearized variables at the equilibrium, we will add the notation $\delta$ to each variable, so $\delta b_{l}(t)=$ $b_{l}(t)-b_{l}^{*}, \delta \dot{b}_{l}=\dot{b}_{l}, \delta \ddot{b}_{l}=\ddot{b}, \delta \bar{b}(t)=\bar{b}(t)-b^{*}, \delta \dot{\bar{b}}=\dot{\bar{b}}, \delta \ddot{\bar{b}}=\ddot{\bar{b}}$, $\dot{\delta \bar{b}}=\ddot{\bar{b}}, \delta \ddot{\tilde{b}}=\ddot{\tilde{b}}, \delta \dot{\tilde{b}}=\dot{\tilde{b}}, \delta \tau_{s}(t)=\tau_{s}(t)-\tau_{s}^{*}, \delta \dot{\tau}_{s}=\dot{\tau}_{s}$, $\delta w_{s}(t)=w_{s}(t)-w_{s}^{*}, \delta \dot{w}_{s}=\dot{w}_{s}, \delta p_{l}(t)=p_{l}(t)-p_{l}^{*}$ and $\delta \dot{p}_{l}=\dot{p}_{l}$.

\section{B. Extended AIMD Model for Multiple Links and Heterogeneous Sources}

The AIMD model for a single link and homogeneous sources in [17], [18] can be extended to the case of multiple links and heterogeneous delays as follows:

$$
\begin{array}{r}
\dot{w}_{s}(t)=\frac{w_{s}\left(t-\tau_{s}^{*}\right)}{\tau_{s}\left(t-\tau_{s}^{*}\right)}\left(1-\sum_{l} R_{l s} p_{l}\left(t-\tau_{l s}^{b *}\right)\right) \frac{1}{w_{s}(t)} \\
-\frac{w_{s}\left(t-\tau_{s}^{*}\right)}{\tau_{s}\left(t-\tau_{s}^{*}\right)} \sum_{l} R_{l s} p_{l}\left(t-\tau_{l s}^{b *}\right) \frac{w_{s}(t)}{2} .
\end{array}
$$

For the purposes of linearization, we note that nonbottleneck links (with empty equilibrium queues) can be ignored. For bottleneck links whose dimension is $L$ in this paper, we make the assumption that rate increase of a source affects all bottlenecks in its path, and write

$$
\dot{b}_{l}(t)=-c_{l}+\sum_{s} R_{l s} \frac{w_{s}\left(t-\tau_{l s}^{f *}\right)}{\tau_{s}\left(t-\tau_{l s}^{f *}\right)} \approx-c_{l}+y_{l}(t) .
$$

Note that $w_{s}(\cdot), b_{l}(\cdot)$, and $p_{l}(\cdot)$ cannot have negative values. But, the present paper does not consider the effect of input/state constraints. $^{2}$

\section{AIMD Model for Single Link and Homogeneous Sources}

From (1) and (2), homogeneous TCP Reno sources with the same window size $\left(d_{s}=d, \tau_{l s}^{f *}=\tau^{f *}, \tau_{l s}^{b *}=\tau^{b *}, w_{s}(t)=\right.$ $w(t)$ ) sharing a common bottleneck router $\left(c_{l}=c, b_{l}(t)=b(t)\right.$, $\left.p_{l}(t)=p(t)\right)$ can be modeled by

$$
\begin{aligned}
\dot{w}(t)=\frac{w\left(t-\tau^{*}\right)}{\left(d+b\left(t-\tau^{*}\right) / c\right)}\left(1-p\left(t-\tau^{b *}\right)\right) \frac{1}{w(t)} \\
-\frac{w\left(t-\tau^{*}\right)}{\left(d+b\left(t-\tau^{*}\right) / c\right)} p\left(t-\tau^{b *}\right) \frac{w(t)}{2}
\end{aligned}
$$

with the queue dynamics

$$
\dot{b}(t)=c+N \frac{w\left(t-\tau^{f *}\right)}{\left(d+b\left(t-\tau^{f *}\right) / c\right)} \approx-c+y(t) .
$$

In [17] and [18], they assume that $\left(w\left(t-\tau^{*}\right) / w(t)\right)(1-p(t-$ $\left.\left.\tau^{b *}\right)\right) \approx 1$ with the dropping probability $p(\cdot)$ which is close to zero, so that their AIMD model is

$$
\dot{w}(t)=\frac{1}{(d+b(t) / c)}-\frac{w\left(t-\tau^{*}\right)}{\left(d+b\left(t-\tau^{*}\right) / c\right)} p\left(t-\tau^{*}\right) \frac{w(t)}{2} .
$$

${ }^{2}$ Refer to [28]-[30] for the design of stabilizing controls in the presence of input/state constraints.

\section{Scope of Our Studies Based on the AIMD Model}

Here, we do not consider the nonlinear dynamic behavior and the effect of input/state constraints (refer to [31] for the chaotic phenomenon of the nonlinear TCP in the presence of constraints). We also do not cover the slow start mode and the fast recovery/fast retransmission mode of TCP (refer to [32] for an initial model and [33] for the performance analysis with the slow start and congestion avoidance modes).

In fact, it seems that applicable theoretical tools are not enough in the sense that implementation of stabilizing controls requires the closed form of explicit solutions instead of sufficient conditions. In this point of view, it will be useful to have a theory telling that which stabilizing control for linearized systems will provide quantitatively better performance for the original nonlinear systems. Note that only the stability relationship between the linearized and original nonlinear systems is known [34].

Nevertheless, we can justify the feedback control design on the linearized system by the objective of feedback control that keeps the closed-loop system around the equilibrium. Note that if all TCP users are far away below equilibrium, they approach the equilibrium point by its increasing mechanism, while AQMs starts to take an action near or above the equilibrium, i.e., in the presence of congestion.

Concluding this subsection, we would like to remark three important issues that we should consider together for the feedback control design: Randomness (refer to some interesting papers based on stochastic models over wired networks [7], [35] and wireless networks [8], [9], [36]; Fairness, where the optimal resource allocation problem has been thoroughly studied in [25] (some associated papers are [37]-[39]); Efficiency, where an initial work is done in [40]. The other issues like quality of service (QoS), cross layering of TCP/IP, and multicast (or overlay) can be considered based on the aforementioned issues.

\section{FeEdBack Control Structure BASED ON A STATE-SPACE MODEL}

The mathematical derivations of this section focus on the case of single link and homogeneous sources with the assumption $\tau^{f}(\cdot)=0$, so that $\tau(t)=\tau^{b}(t)$ (We will study how to relax this assumption in Section IV-B).

In Section III-A, we derive a state-space model which leads to PD-type state-feedback control in terms of queue length or RTT. In Section III-B, we will extend this procedure in the presence of an additional dynamics like a low-pass filter of RED. A mathematical framework is proposed in Section III-C as an attempt to interpret existing AQM algorithms in a unified manner which will be discussed in detail in Section V.

\section{A. State-Feedback Control}

The first key step to deriving a state-feedback control structure is to convert two coupled dynamics (2) and (3) to the equiv- 
alent single dynamical system by differentiating (3) and rearranging the differentiated equation with (2) and (3)

$$
\begin{aligned}
\ddot{b}(t)= & \frac{N\left(\dot{b}\left(t-\tau^{*}\right)+c\right)}{(d+b(t) / c)^{2}(\dot{b}(t)+c)}-\frac{(\dot{b}(t)+c) \dot{b}(t)}{(d+b(t) / c) c} \\
- & \left\{\frac{N\left(\dot{b}\left(t-\tau^{*}\right)+c\right)}{(d+b(t) / c)^{2}(\dot{b}(t)+c)}\right. \\
& \left.+\frac{\left(\dot{b}\left(t-\tau^{*}\right)+c\right)(\dot{b}(t)+c)}{2 N}\right\} p\left(t-\tau^{*}\right) \\
= & f\left(b(t), \dot{b}(t), \dot{b}\left(t-\tau^{*}\right), p\left(t-\tau^{*}\right)\right) .
\end{aligned}
$$

In general, it is difficult to systematically find a nonlinear function $p(t)$ which guarantees the global asymptotic stability for nonlinear dynamical systems with delays (see the equivalent nonlinear system (33) for multiple links and heterogeneous sources). As a starting point to address these problems and as a method to avoid the nonnegative constraint, the present paper considers an equilibrium point with positive values and studies the linearized system of (5) on $b(t), \dot{b}(t), \dot{b}\left(t-\tau^{*}\right)$, and $p\left(t-\tau^{*}\right)$ near the equilibrium point.

From (5), we can derive the following model of the linearized TCP and queue dynamics:

$$
\delta \ddot{b}(t)=A_{1} \delta b(t)+A_{2} \delta \dot{b}(t)+B_{1} \delta p\left(t-\tau^{*}\right)
$$

where $\delta b(0), \delta \dot{b}(0)$ and $\left\{\delta p(\sigma), \sigma \in\left[-\tau^{*}, 0\right]\right\}$ are given, $A_{1}=-\left(2 c N / \tau^{*}\left(2 N^{2}+c^{2} \tau^{* 2}\right)\right), A_{2}=-\left(\left(2 c N \tau^{*}+\right.\right.$ $\left.\left.2 N^{2}+c^{2} \tau^{* 2}\right) / \tau\left(2 N^{2}+c^{2} \tau^{* 2}\right)\right)$, and $B_{1}=-\left(\left(2 N^{2}+\right.\right.$ $\left.\left.c^{2} \tau^{* 2}\right) / 2 \tau^{* 2} N\right)$. Refer to Appendix I for derivation of (6). For presentation of the linearized variables, refer to the end of Section II-A.

The differential equation (6) can be represented as the following state-space model:

$$
\dot{z}(t)=A z(t)+B \delta p\left(t-\tau^{*}\right)
$$

where

$$
z(t)=\left[\begin{array}{c}
\delta b(t) \\
\delta \dot{b}(t)
\end{array}\right] \quad A=\left[\begin{array}{cc}
0 & 1 \\
A_{1} & A_{2}
\end{array}\right] \quad B=\left[\begin{array}{c}
0 \\
B_{1}
\end{array}\right] .
$$

The previous state-space model is a minimal representation with state variables $(\delta b(t), \delta \dot{b}(t))$ of (6). Note that the open-loop system of (7) (i.e., $\dot{z}(t)=A z(t)$ ) is asymptotically stable since its system matrix $A$ has negative eigenvalues $\left(A_{2}+\sqrt{A_{2}^{2}+4 A_{1}}\right) / 2$ and $\left(A_{2}-\sqrt{A_{2}^{2}+4 A_{1}}\right) / 2$, which means that the feedback control $\delta p(\cdot)=0$ (i.e, $p(\cdot)=p^{*}$ ) makes the system stable under the assumption of $\tau^{f}(\cdot)=0 .{ }^{3}$

From the above state-space model, we can naturally get a PD-type state-feedback control

$$
\delta p(t)=H z(t)=H_{P} \delta b(t)+H_{D} \delta \dot{b}(t)
$$

3In this paper, "stable" means "asymptotically stable," not "marginally stable" which corresponds to "oscillating." if we ignore the time delay $\tau^{*}$ (i.e., $\tau^{*}=0$ ) in the control $\delta p\left(t-\tau^{*}\right)$. How to deal with the delay and how to obtain a pair of a stabilizing gain $\left(H_{P}, H_{D}\right)$ will be discussed in Section IV and Section V, respectively.

The derived PD-type control structure, which we also did not expect, is interesting in the following sense: It supports the conjecture of the networking literature in terms of the feedback control structure for the first time to our knowledge that AQM RED is not enough to regulate the given TCP [12], [14], where RED can be classified as a P-type AQM (i.e., $H_{D}=0$ ) or as an output feedback control. When we ignore the time delay $\tau^{*}$, the difference between the state- and output-feedback controls is as follows. With P-type RED with $H_{D}=0$, the closed-loop system (7) is

$$
\dot{z}(t)=\left[\begin{array}{cc}
0 & 1 \\
A_{1}+B_{1} H_{P} & A_{2}
\end{array}\right] z(t)
$$

while the closed-loop system with (9) is

$$
\dot{z}(t)=\left[\begin{array}{cc}
0 & 1 \\
A_{1}+B_{1} H_{P} & A_{2}+B_{1} H_{D}
\end{array}\right] z(t) .
$$

From these equations, it is easy to see that we cannot adjust eigenvalues of the closed-loop system arbitrarily with the output feedback control, while we can do that with the state-feedback control. Thus, the advantage of the state-feedback control comes from having the same degree of freedom as that of the system, while the output-feedback control has a less one. The case of REM/PI, which adds an integral control action to RED, will be discussed in Section III-C.

Remark 1: In order to relate the derived structure with the previous ones based on the transfer function approach, we consider the transfer function from $\delta b(t)$ to $\delta w(t)$ for the linearized system of (2) $\left(\delta \dot{w}(t)=-k_{1} \delta w(t)+k_{2} \delta p(t)\right)$ with control (9). Then, the resulting transfer function is equal to the lead-lag compensator [41] with the form $\delta w(s) / \delta b(s)=k_{2}\left(H_{P}+H_{D} s\right) /\left(s+k_{1}\right)$. As mentioned in [3, Rem. 1], the feedback control structures in Vegas [42] and a new static TCP [43] can be considered as P-type controls in terms of RTT or queue length (i.e., $H_{D}=0$ ). Note that [44] and [45] add D-type control to [42] and [43], respectively, after our papers [1], [2] have shown that the PD-type control is a natural state-feedback structure to stabilize the given AIMD model.

Remark 2: The PD-type structure obtained in this paper can also be implemented at sources as follows. Similarly to (5), using $\tau(t)=d+b(t) / c$, (2) and (3) can be converted to the equivalent single dynamical system: $\ddot{\tau}(t)=\left(N\left(\dot{\tau}\left(t-\tau^{*}\right)+\right.\right.$ 1) $\left./(d+b(t) / c)^{2}(c \dot{\tau}(t)+c)\right)-((\dot{\tau}(t)+1) \dot{\tau}(t) /(d+b(t) / c))-$ $\left\{\left(N\left(\dot{\tau}\left(t-\tau^{*}\right)+1\right) /(d+b(t) / c)^{2}(c \dot{\tau}(t)+c)\right)+\left(\left(\dot{\tau}\left(t-\tau^{*}\right)+\right.\right.\right.$ 1) $(c \dot{\tau}(t)+c) / 2 N\} p\left(t-\tau^{*}\right)$. From this equivalent form, the state-space model of the linearized TCP and queue dynamics is given by (7) and (8) with $z(t)$ and $B_{1}$ replaced by $z(t)=\left[\begin{array}{l}\delta \tau(t) \\ \delta \dot{\tau}(t)\end{array}\right]$ and $B_{1}=-\left(\left(2 N^{2}+c^{2} \tau^{* 2}\right) / 2 c \tau^{* 2} N\right)$, respectively. From 
this state-space model, we can naturally get another PD-type state-feedback control in terms of RTT

$$
\delta p(t)=H z(t)=H_{P} \delta \tau(t)+H_{D} \delta \dot{\tau}(t)
$$

if we ignore the time delay $\tau^{*}$ (i.e., $\tau^{*}=0$ ) in the control $\delta p\left(t-\tau^{*}\right)$. Thus, our design procedures and control structures based on the state $\delta b(t)$ and $\delta \dot{b}(t)$ hold.

Stabilized Vegas in [44], which was called FAST (Fast AQM and Scalable TCP) protocol, assumes the fixed RTT when they linearize the original nonlinear model to get the stabilizing gains of the PD-type feedback control structure. It is natural that the fixed RTT gives a conservative stability condition in the presence of queue dynamics, where our works consider the timevarying RTT.

For implementation, (9) can be rewritten as the equivalent form $p(t)=p^{*}+H_{P}\left(b(t)-b^{*}\right)+H_{D}(y(t)-c)$. Thus, our PD-type structure can be considered as combination of queue and rate controls. Since the AIMD model (2) is developed under the assumption that the congestion measure $p(t)$ is small, the equilibrium probability $p^{*}$ should be small if dropping is used as the congestion measure. A high $p^{*}$ can cause frequent retransmissions and timeouts which lead to the mismatch between the AIMD model and the congestion mode of TCP. However, it can be large if marking like explicit congestion notification (ECN) is used as the congestion measure [46] which will not be studied in this paper. $p^{*}$ should also not be so close to zero in order not to make $p(t)$ saturated, where saturation can be a cause of instability or chaos as shown in [31]. For the same reason, $b^{*}$ should not be so close to zero. Selection of $b^{*}$ should consider two more things. First, a very large queueing delay can make the nominal-stable system oscillate if the delay is not compensated appropriately. Second, small packets (mice) or user datagram protocol (UDP) flows do not interact with the congestion information which can be considered as noise or disturbance for the dynamical model (2). ${ }^{4}$ Thus, $b^{*}$ should be selected so that non-reacting packets to the congestion information go through networks without causing congestion. If $b^{*}$ is very small or closed to the maximum queue size, it is not easy to stabilize system (2) with (9) because of the input/state constraints.

In this subsection, we derived the state-space model and its state-feedback control structure, where the derived PD-type feedback control implies the structural deficiency of P-type RED. However, this subsection did not consider average queueing dynamics of RED which is used to make the dynamic behavior of TCP smooth for the burst traffic [10]. This case is studied in the following subsection.

\section{B. With Additional Dynamics: Low-Pass Filter of RED}

Consider the dynamics of average queueing $\bar{b}(t)$ in RED which is called the low-pass filter in the control literature

$$
\dot{\bar{b}}(t)=-P_{1} \bar{b}(t)+P_{1} b(t) \quad P_{1}>0 .
$$

${ }^{4}$ For one way to model and deal with the disturbance based on the state-space approach, see [47].
Here, $P_{1}$ is a design parameter which decides the cut-off frequency [41]. From (6) and (11), we can get

$$
\delta \dddot{\bar{b}}(t)=\bar{A}_{2} \delta \ddot{\bar{b}}(t)+\bar{A}_{1} \delta \dot{\bar{b}}(t)+\bar{A}_{3} \delta \bar{b}(t)+P_{1} B_{1} \delta p\left(t-\tau^{*}\right)
$$

where $\bar{A}_{1}=A_{1}+A_{2} P_{1}, \bar{A}_{2}=A_{2}-P_{1}, \bar{A}_{3}=A_{1} P_{1}$. The differential equation (12) can be represented as the following state-space model:

$$
\dot{\bar{z}}(t)=\bar{A} \bar{z}(t)+\bar{B} \delta p\left(t-\tau^{*}\right)
$$

with $\bar{z}(0)$ and $\left\{\delta p(\sigma), \sigma \in\left[-\tau^{*}, 0\right]\right\}$, where

$\bar{z}(t)=\left[\begin{array}{c}\delta \bar{b}(t) \\ \delta \dot{\bar{b}}(t) \\ \dot{\bar{b}}(t)\end{array}\right] \quad \bar{A}=\left[\begin{array}{ccc}0 & 1 & 0 \\ 0 & 0 & 1 \\ \bar{A}_{3} & \bar{A}_{1} & \bar{A}_{2}\end{array}\right] \quad \bar{B}=\left[\begin{array}{c}0 \\ 0 \\ P_{1} B_{1}\end{array}\right]$.

From the previous state-space model, we can naturally get the PD-type state-feedback control in the presence of a low-pass filter

$$
\delta p(t)=\bar{H} z(t)=\bar{H}_{1} \delta \bar{b}(t)+\bar{H}_{2} \delta \dot{\bar{b}}(t)+\bar{H}_{3} \delta \ddot{\bar{b}}(t)
$$

if we ignore the time delay $\tau^{*}$. Note that RED with a low-pass filter can be represented as $\delta p(t)=\bar{H}_{1} \delta \bar{b}(t)$, where the derived structure (14) also shows that RED is not enough to fully control TCP dynamics of the AIMD model.

For implementation, (14) can be rewritten using (3) and (11) as the equivalent form $p(t)=p^{*}+\bar{H}_{1}\left(\bar{b}(t)-b^{*}\right)+$ $\bar{H}_{2}\left(-P_{1} \hat{b}(t)+P_{1} b(t)\right)-\bar{H}_{3} P_{1}\left(-P_{1} \hat{b}(t)+P_{1} b(t)\right)+$ $\bar{H}_{3} P_{1}(y(t)-c)$, which does not require the estimation of $\dot{\bar{b}}(t)$ and $\ddot{\bar{b}}(t)$.

In this subsection, we showed how to design a state-feedback control in the presence of a low-pass filter. In fact, the results in Section III-A can also be applied to other cases of additional dynamics. In the following subsection, we derive a state-feedback control in the presence of integral control action.

\section{A Unified Mathematical Framework Using Integral Control Action}

This subsection applies integral control action technique in [48] to the system (7) as a trial to interpret other AQM algorithms such as REM and PI in a unified mathematical framework rather than to compare them via simulation, where impact of each structure on performance will be discussed in Section V. Similarly to REM and PI, this subsection does not consider the low-pass filter of RED for ease of discussion.

The key step to applying the technique is to have another derivative of system (2) as follows:

$$
\begin{aligned}
\dddot{b}(t)= & \frac{\partial f}{\partial b(t)} \dot{b}(t)+\frac{\partial f}{\partial \dot{b}(t)} \ddot{b}(t)+\frac{\partial f}{\partial \dot{b}\left(t-\tau^{*}\right)} \ddot{b}\left(t-\tau^{*}\right) \\
& +\frac{\partial f}{\partial p\left(t-\tau^{*}\right)} \dot{p}\left(t-\tau^{*}\right) \\
= & g\left(b(t), \dot{b}(t), \ddot{b}(t), \dot{b}\left(t-\tau^{*}\right), \ddot{b}\left(t-\tau^{*}\right),\right. \\
& \left.p\left(t-\tau^{*}\right), \dot{p}\left(t-\tau^{*}\right)\right) .
\end{aligned}
$$


From (15), we can derive the following third-order linearized AIMD model:

$$
\dot{z}_{e}(t)=A_{e} z_{e}(t)+B_{e} \delta \dot{p}\left(t-\tau^{*}\right)
$$

where $z_{e}(0)$ and $\left\{\delta \dot{p}(\sigma), \sigma \in\left[-\tau^{*}, 0\right]\right\}$ are given, $z_{e}(t)=$ $\left[\begin{array}{c}z_{0}(t) \\ \dot{z}(t)\end{array}\right], A_{e}=\left[\begin{array}{cc}0 & I_{e} \\ 0 & A\end{array}\right], B_{e}=\left[\begin{array}{l}0 \\ B\end{array}\right]$ with $z(t), A$ and $B$ in (8), $z_{0}(t)=I_{e} z(t)$, and $I_{e}=[1,0]$. Refer to Appendix I for derivation of (16). It is easy to check that the pair $\left(A_{e}, B_{e}\right)$ is stabilizable if $B_{1} \neq 0$.

From the previous state-space model, we can naturally get a PID-type ${ }^{5}$ state-feedback control

$$
\delta \dot{p}(t)=H_{e} z_{e}(t)=H_{I} \delta b(t)+H_{P} \delta \dot{b}(t)+H_{D} \delta \ddot{b}(t)
$$

if we ignore the time delay $\tau^{*}$. As discussed in Section III-A, setting $H_{P}=0$ or $H_{D}=0$ has a limitation on adjusting closedloop eigenvalues, i.e., on controlling the closed-loop dynamic behavior. The implication of $H_{I}=0$ is that the augmented system (16) can be reduced to the original second-order system (7).

For implementation, (17) can be rewritten as the equivalent form $p(t)=p\left(t_{0}\right)+H_{I} \int_{t_{0}}^{t}\left(b(\sigma)-b^{*}\right) d \sigma+H_{P}(b(t)-$ $\left.b\left(t_{0}\right)\right)+H_{D}\left(y(t)-y\left(t_{0}\right)\right)$. Note that the PID-type feedback control does not require the equilibrium point $p^{*}$ even for the case that $H_{I}=0$, while the PD-type feedback control needs $p^{*}$.

Now, we discuss the implication of integral action on the closed-loop dynamics.

First, we consider its effect at the steady state. Without integral action, the stabilizing feedback control $\delta p(t)$ makes $\|z(t)\|$ approach zero which in turn makes $\|\delta p(t)\|$ approach zero since $\delta p(t)=H z(t)$. As a result, the small $\delta p(t)$ makes $\|z(t)\|$ approach zero slowly, although this effect may be marginal for the global performance. With integral action, the resulting feedback control $\delta \dot{p}(t)=H_{e} z_{e}(t)$ at the time $t$ reflects the accumulation of $\left(b(\sigma)-b^{*}\right)$ additionally, i.e., $p(t)=p\left(t_{0}\right)+H_{e} \int_{t_{0}}^{t} z_{e}(\sigma) d \sigma=$ $p\left(t_{0}\right)+H_{I} \int_{t_{0}}^{t}\left(b(\sigma)-b^{*}\right) d \sigma+H\left(z(t)-z\left(t_{0}\right)\right)$ which makes the steady-state tracking error of $z(t)$ approach zero faster.

In the same way, at the transient state, this action makes the system approach the equilibrium faster when the queue $b(t)$ and rate $y(t)$ are below the equilibrium point since $H_{I}$ is non-negative and $H_{D}$ is positive for stability as shown in Section V. However, since the integral structure accumulates the previous state information, it can make the system go over the equilibrium point easily, i.e., cause an overshoot. Then, the system goes back slowly to the equilibrium due to the damping property of the integral action. If the overshoot exceeds the maximum queue size, it causes a windup phenomenon due to the saturation which can severely degrade the performance (For antiwindup techniques, refer to [50] and [51]). Derivative structure of the state-feedback controls reduces this damping phenomenon and makes the system go and back to the equilibrium fast. This is another main reason why we need the PID-type state-feedback control instead

${ }^{5}$ We have noticed that inner and outer loop PI-type AQM to the aggregate rate in [21] corresponds to the PID-type delay-independent AQM to the real queue length. Another delay-independent PID-type AQM is proposed in [49]. of PI-type REM/PI, where REM/PI can be classified as output feedback controls like P-type RED.

Until now, we ignored the time delay in the control (congestion measure), i.e., we did not compensate for delays. Let's assume that we use $\delta p(t)=H z(t)$ in (9) for the delayed system (7) [or (17) for (16)]. This kind of controls including RED, REM, PI, AVQ, and AQMs in [21] and [22] are called delay-independent (or memoryless) controls in the literature. Then, the closed-loop system is given by $\dot{z}(t)=A z(t)+B H z\left(t-\tau^{*}\right)$ (or $\dot{z}_{e}(t)=A_{e} z_{e}(t)+B_{e} H_{e} z_{e}\left(t-\tau^{*}\right)$ ) and thus has infinite number of eigenvalues. As the delay value $\tau^{*}$ increases over some finite value (depending on $A, B$ and $H$ ), the number of positive eigenvalues increases, i.e., the closed-loop system oscillates. The way to solve this problem is to have a small control gain $H$ as done in [21] and [22] or setting $H=0$ so that the system (7) with $\delta p(\cdot)=0$ dominates, where the system (7) with $\delta p(\cdot)=0$ is asymptotically stable under the assumption $\tau^{f}(\cdot)=0$. With the small control gain, however, the closed-loop system cannot approach the equilibrium much faster than the system (7) with $\delta p(\cdot)=0$, i.e., delay-independent controls cannot regulate the delayed dynamics arbitrarily in the presence of large delays. This phenomenon is much more severe with the output-feedback control. This is the main reason why we need to develop a delay-dependent control which compensates for delays explicitly. The following section investigates how to design a delay-dependent control that uses not only the current dynamic information at time $t$ but also the accumulated control information from $t-\tau^{*}$ to $t$. Note that the integral control in REM/PI, which use the accumulated state information, is not constructed for the delay compensation.

\section{Derivation of the Delay-DePendent STATE-FEEDBACK CONTROL}

First, we propose how to compensate for the delay in the congestion measure explicitly under the same assumption of Section III that the forward delay from source to router is zero. This assumption is relaxed by applying a modified virtual queue dynamics in the second subsection. For simplicity of notation, throughout the rest of this paper, we define $e_{1}=e^{-a_{1} \tau^{*}}-e^{-a_{2} \tau^{*}}$, $e_{2}=a_{1} e^{-a_{1} \tau^{*}}-a_{2} e^{-a_{2} \tau^{*}}, e_{3}=a_{2} e^{-a_{1} \tau^{*}}-a_{1} e^{-a_{2} \tau^{*}}$, $e_{4}=-\left(1 / e_{1}\right)\left[\left(a_{1} / a_{2}\right) e^{-a_{1} \tau^{*}}-\left(a_{2} / a_{1}\right) e^{-a_{2} \tau^{*}}+\left(\left(a_{2}^{2}-a_{1}^{2}\right) /\right.\right.$ $\left.\left.a_{1} a_{2}\right) e^{-A_{2} \tau^{*}}\right], e_{5}=\left(1 / e_{1}\right)\left[\left(1 / a_{2}\right) e^{-a_{1} \tau^{*}}-\left(1 / a_{1}\right) e^{-a_{2} \tau^{*}}+\right.$ $\left.\left(\left(a_{2}-a_{1}\right) / a_{1} a_{2}\right) e^{-A_{2} \tau^{*}}\right], a_{1}=A_{2}+\sqrt{A_{2}^{2}+4 A_{1}} / 2$, $a_{2}=\left(A_{2}-\sqrt{A_{2}^{2}+4 A_{1}}\right) / 2, \hat{B}_{1}=B_{1}\left(a_{2}-a_{1}\right) e^{-A_{2} \tau^{*}} / e_{1}$.

\section{A. Compensation for the Delay in Feedback Control}

The key to deriving an explicit memory control for the delayed system (7) is to transform the delayed system (7) to the equivalent nominal system

$$
\dot{s}(t)=A s(t)+\hat{B} \delta p(t)
$$

where $s(t)=\left[s_{1}(t), s_{2}(t)\right]^{T}, \hat{B}=\left[0, \hat{B}_{1}\right]^{T}$ with $\hat{B}_{1}$, $s_{1}(t)=-\left(e_{2} / e_{1}\right)\left(\delta b(t)+u_{1 \tau}(t)\right)+\left(\delta \dot{b}(t)+u_{2 \tau}(t)\right), s_{2}(t)=$ $A_{1}\left(\delta b(t)+u_{1 \tau}(t)\right)+\left(e_{3} / e_{1}\right)\left(\delta \dot{b}(t)+u_{2 \tau}(t)\right),\left[\begin{array}{l}u_{1 \tau}(t) \\ u_{2 \tau}(t)\end{array}\right]=$ $\left(B_{1} /\left(a_{1}-a_{2}\right)\right) \int_{-\tau^{*}}^{0}\left[\begin{array}{c}e^{-\left(\sigma+\tau^{*}\right) a_{1}}-e^{-\left(\sigma+\tau^{*}\right) a_{1}}-a_{2} e^{-\left(\sigma+\tau^{*}\right) a_{2}} \\ a^{-\left(\sigma+\tau^{*}\right.}\end{array}\right] \delta p(\sigma+t) d \sigma$. 
Refer to Appendix II for derivation of (18). It is easy to see that if $\tau^{*}=0$, then $s(t)=z(t)$ and $\hat{B}=B$. Note that the pair $(A, \hat{B})$ is stabilizable, and the closed-loop system of (18) is asymptotically stable if and only if the transformed system (7) is asymptotically stable. Since $A_{2}^{2}+4 A_{1}>0$ for system matrices of (7), we have $a_{1} \neq a_{2} \neq 0, e_{1}<0$ and $\hat{B}_{1}<0$ for $\tau^{*}>0$, while the linearized model of [17, eq. (4)], [18] has $a_{1}=a_{2}$ when $2 N=\tau^{*} c$ even for $\tau^{*}>0$.

Thus, by adding the memory control structure $u_{1 \tau}(t)$ and $u_{2 \tau}(t)$, we can handle the delay in congestion measure explicitly. From the previous state-space model, we can get a PD-type delay-dependent state-feedback control

$$
\delta p(t)=H_{P}^{\tau}\left(\delta b(t)+u_{1 \tau}(t)\right)+H_{D}^{\tau}\left(\delta \dot{b}(t)+u_{2 \tau}(t)\right) .
$$

Equivalently, it can be rewritten as $p(t)=p^{*}+H_{P}^{\tau}\left(b(t)-b^{*}+\right.$ $\left.u_{1 \tau}(t)\right)+H_{D}^{\tau}\left(y(t)-c+u_{2 \tau}(t)\right)$.

Similarly, (16) can be transformed to the equivalent nominal system

$$
\dot{s}_{e}(t)=A_{e} s_{e}(t)+\hat{B}_{e} \delta \dot{p}(t)
$$

where $s_{e}(t)=\left[s_{1}(t), s_{2}(t), s_{3}(t)\right]^{T}, \hat{B}_{e}=\left[0, \hat{B}^{T}\right]^{T}$

$$
\begin{aligned}
s_{1}(t)= & \left(a_{2}-a_{1}\right) e^{-A_{2} \tau^{*}} / e_{1}\left(\delta b(t)+\dot{u}_{1 \tau}(t)\right) \\
& +e_{4}\left(\delta \dot{b}(t)+\dot{u}_{2 \tau}(t)\right)+e_{5}\left(\delta \ddot{b}(t)+\dot{u}_{3 \tau}(t)\right) \\
s_{2}(t)= & -e_{2} / e_{1}\left(\delta \dot{b}(t)+\dot{u}_{2 \tau}(t)\right)+\left(\delta \ddot{b}(t)+\dot{u}_{3 \tau}(t)\right) \\
s_{3}(t)= & A_{1}\left(\delta \dot{b}(t)+\dot{u}_{2 \tau}(t)\right)+e_{3} / e_{1}\left(\delta \ddot{b}(t)+\dot{u}_{3 \tau}(t)\right)
\end{aligned}
$$

$$
\begin{aligned}
{\left[\begin{array}{c}
\dot{u}_{1 \tau}(t) \\
\dot{u}_{2 \tau}(t) \\
\dot{u}_{3 \tau}(t)
\end{array}\right]=} & \frac{B_{1}}{\left(a_{1}-a_{2}\right)} \\
& \times \int_{-\tau^{*}}^{0}\left[\begin{array}{c}
\frac{\left(a_{1}-a_{2}\right)}{a_{1} a_{2}}+\frac{e^{-\left(\sigma+\tau^{*}\right) a_{1}}}{a_{1}}-\frac{e^{-\left(\sigma+\tau^{*}\right) a_{2}}}{\left.e^{-\left(\sigma+\tau^{*}\right.}\right) a_{1}}-e^{-\left(\sigma+\tau^{*}\right) a_{2}} \\
a_{1} e^{-\left(\sigma+\tau^{*}\right) a_{1}}-a_{2} e^{-\left(\sigma+\tau^{*}\right) a_{2}}
\end{array}\right] \\
& \times \delta \dot{p}(\sigma+t) d \sigma .
\end{aligned}
$$

Refer to Appendix II for derivation of (20). The pair $\left(A_{e}, \hat{B}_{e}\right)$ is stabilizable (or controllable) if the pair $(A, B)$ is stabilizable (or controllable).

From the previous model, we can get a PID-type delay-dependent state-feedback control

$$
\begin{aligned}
\delta \dot{p}(t)=H_{I}^{\tau}\left(\delta b(t)+\dot{u}_{1 \tau}(t)\right)+ & H_{P}^{\tau}\left(\delta \dot{b}(t)+\dot{u}_{2 \tau}(t)\right) \\
& +H_{D}^{\tau}\left(\delta \ddot{b}(t)+\dot{u}_{3 \tau}(t)\right) .
\end{aligned}
$$

The equivalent form is $p(t)=p\left(t_{0}\right)+H_{I}^{\tau}\left(\int_{t_{0}}^{t}\left(b(\sigma)-b^{*}\right) d \sigma+\right.$ $\left.u_{1 \tau}(t)\right)+H_{P}^{\tau}\left(b(t)-b\left(t_{0}\right)+u_{2 \tau}(t)\right)+H_{D}^{\tau}\left(y(t)-y\left(t_{0}\right)+u_{3 \tau}(t)\right)$, where

$$
\begin{aligned}
& {\left[\begin{array}{l}
u_{1 \tau}(t) \\
u_{2 \tau}(t) \\
u_{3 \tau}(t)
\end{array}\right]=\left(B_{1} /\left(a_{1}-a_{2}\right)\right)} \\
& \qquad \int_{-\tau^{*}}^{0}\left[\begin{array}{c}
\frac{\left(a_{1}-a_{2}\right)}{a_{1} a_{2}}+\frac{e^{-\left(\sigma+\tau^{*}\right) a_{1}}}{a_{1}}-\frac{e^{-\left(\sigma+\tau^{*}\right) a_{2}}}{a_{2}} \\
e^{-\left(\sigma+\tau^{*}\right) a_{1}}-e^{-\left(\sigma+\tau^{*}\right) a_{2}} \\
a_{1} e^{-\left(\sigma+\tau^{*}\right) a_{1}}-a_{2} e^{-\left(\sigma+\tau^{*}\right) a_{2}}
\end{array}\right] \\
& \left(p(\sigma+t)-p\left(\sigma+t_{0}\right)\right) d \sigma .
\end{aligned}
$$

The procedures in this subsection can directly be applied to the case of a low-pass filter which has one more dimension as shown in Section III-B. For more details, refer to [4].

Next, we propose how to relax the assumption made in the derivation of (5) that the forward delay from source to router is zero, still compensating for delays explicitly.

\section{B. Arbitrary Delay Compensation Based on a Modified Virtual Queue}

The forward delay $\tau_{s}^{f}(t)$ from source to router produces a state-delay for the linearized system of the coupled dynamics (2) and (3) that makes it impossible to compensate for delays in a closed-form since the state-delayed system is infinite-dimensional (infinite number of eigenvalues).

In order to overcome this problem, we add the following modified virtual queue dynamics $(\dot{\tilde{b}}(t))$ at the router6 to the AIMD model (2)

$$
\dot{\tilde{b}}(t)=\gamma_{1}\left(-\gamma_{2} c+N \frac{w\left(t-\tau^{f *}\right)}{\tau\left(t-\tau^{f *}\right)}\right) \approx \gamma_{1}\left(-\gamma_{2} c+y(t)\right)
$$

where $\tilde{b}(t)$ is the virtual queue length, and $\gamma_{1}(>0)$ and $\gamma_{2}$ are free design parameters.

If $0<\gamma_{2}<1, N\left(w^{*} / \tau^{*}\right)=\gamma_{2} c$ and $b^{*}=0$. Hence, we have $\tau^{*}=\tau^{f *}+\tau^{b *}=d, w^{*}=(d / N) \gamma_{2} c$, and $p^{*}=2 /\left(2+w^{* 2}\right)=$ $2 N^{2} /\left(2 N^{2}+d^{2} \gamma_{2}^{2} c^{2}\right)$

Since the virtual queue dynamics is dominant near the equilibrium point, we approximate that the real queue $b(\cdot)$ is zero near the operating point in this subsection thus, the round trip time $\tau\left(t-\tau^{f *}\right)$ is approximated as the propagation delay $d$.

Then, (2) based on the aforementioned virtual queue dynamics is converted to the equivalent form $\ddot{\tilde{b}}(t)=$ $\left(\gamma_{1} N\left(\dot{\tilde{b}}(t-d)+\gamma_{1} \gamma_{2} c\right) / d^{2}\left(\dot{\tilde{b}}(t)+\gamma_{1} \gamma_{2} c\right)\right)-\left\{\left(\gamma_{1} N(\dot{\tilde{b}}(t-\right.\right.$ $\left.\left.d)+\gamma_{1} \gamma_{2} c\right) / d^{2}\left(\dot{\tilde{b}}(t)+\gamma_{1} \gamma_{2} c\right)\right)+\left(\left(\dot{\tilde{b}}(t-d)+\gamma_{1} \gamma_{2} c\right)(\dot{\tilde{b}}(t)+\right.$ $\left.\left.\left.\gamma_{1} \gamma_{2} c\right) / 2 \gamma_{1} N\right)\right\} p(t-d)$.

Similarly to the previous sections, its linearized state-space model is given by

$$
\delta \ddot{\tilde{b}}(t)=\tilde{A}_{2} \delta \dot{\tilde{b}}(t)+\tilde{B}_{1} \delta p(t-d)
$$

where $\delta \dot{\tilde{b}}(0)$ and $\{\delta p(\sigma), \sigma \in[-d, 0]\}$ are given, $\tilde{A}_{2}=$ $-\left(2 \gamma_{2} c N /\left(2 N^{2}+\gamma_{2}^{2} c^{2} d^{2}\right)\right)$, and $\tilde{B}_{1}=-\left(\gamma_{1}\left(2 N^{2}+\right.\right.$ $\left.\left.\gamma_{2}^{2} c^{2} d^{2}\right) / 2 d^{2} N\right)$.

Note that the previous linearized model does not include the term $\dot{\tilde{b}}(t-d)$. This interesting and nonintuitive property allows us to use the same delay compensation technique as that in the previous subsection.

The state-space model (22) leads to the following $P$-type state-feedback control in terms of aggregate

$$
\delta p(t)=\tilde{H}_{P}\left(\delta \dot{\tilde{b}}(t)+u_{1 d}(t)\right)
$$

where $u_{1 d}(t)=\int_{-d}^{0} e^{-\tilde{A}_{2}(\sigma+d)} \tilde{B}_{1} \delta p(\sigma+t) d \sigma$ [or $p(t)=p^{*}+$ $\left.\left.\tilde{H}_{P}\left(\gamma_{1}\left(-\gamma_{2} c+y(t)\right)+u_{1 d}(t)\right)\right)\right]$.

${ }^{6}$ If $\gamma_{1}=\gamma_{2}=1$, the virtual queue dynamics is equal to the real queue dynamics, and the AVQ in [20] assumes $\gamma_{1}<0$ while we assumes $\gamma_{1}>0$ in this paper. 
It is interesting to see that the delay-independent AVQ in [15], [20] seems to be a $P$-type control in terms of aggregate since linearizing the AVQ $p(t)=p(\dot{\tilde{b}}(t))$ gives $\delta p(t)=\tilde{H}_{P}^{a} \delta \dot{\tilde{b}}(t)$, which is the same as (23) in the absence of the delay $d$. Thus, our result verifies that the simplified AVQ is the delay-independent state-feedback control for the AIMD model when the virtual queue dynamics is dominant.

Similarly to Section III-C, if one wants to make the steadystate tracking error approach zero fast, we can derive the extended state-space model with integral control action

$$
\dot{\tilde{z}}(t)=\tilde{A} \tilde{z}(t)+\tilde{B} \delta \dot{p}(t-d)
$$

where $\tilde{z}(0)$ and $\{\delta \dot{p}(\sigma), \sigma \in[-d, 0]\}$ are given, $\tilde{z}(t)=\left[\begin{array}{c}\delta \dot{\tilde{b}}(t) \\ \tilde{\delta} \tilde{\tilde{b}}(t)\end{array}\right]$, $\tilde{A}=\left[\begin{array}{cc}0 & 1 \\ 0 & \tilde{A}_{2}\end{array}\right], \tilde{B}=\left[\begin{array}{c}0 \\ \tilde{B}_{1}\end{array}\right]$.

The equivalent nominal system of the delayed system (24) is given by

$$
\dot{\tilde{s}}_{e}(t)=\tilde{A} \tilde{s}_{e}(t)+\hat{\tilde{B}} \delta \dot{p}(t)
$$

where $\tilde{s}_{e}(t)=\left[\tilde{s}_{1}(t), \tilde{s}_{2}(t)\right]^{T}, \hat{\tilde{B}}=\left[0, \hat{\tilde{B}}_{1}\right]^{T}, \tilde{s}_{1}(t)=$ $\left(\tilde{A}_{2} e^{-\tilde{A}_{2} d} /\left(1-e^{-\tilde{A}_{2} d}\right)\right)\left(\delta \tilde{b}(t)+\dot{u}_{1 d}(t)\right)+\left(\delta \tilde{\tilde{b}}(t)+\dot{u}_{2 d}(t)\right)$, $\tilde{s}_{2}(t)=\left(\tilde{A}_{2} /\left(1-e^{-\tilde{A}_{2} d}\right)\right)\left(\dot{\tilde{\tilde{b}}}(t)+\dot{u}_{2 d}(t)\right)$, $\tilde{\tilde{B}}_{1}=\tilde{B}_{1} \tilde{A}_{2} e^{-\tilde{A}_{2} d} /\left(1-e^{-\tilde{A}_{2} d}\right), \quad\left[\begin{array}{l}\dot{u}_{1 d}(t) \\ u_{2 d}(t)\end{array}\right]=$ $-\left(\tilde{B}_{1} / \tilde{A}_{2}\right) \int_{-d}^{0}\left[\begin{array}{c}1-e^{-(\sigma+d)} \tilde{A}_{2} \\ -\tilde{A}_{2} e^{-(\sigma+d)} \tilde{A}_{2}\end{array}\right] \delta \dot{p}(\sigma+t) d \sigma, \quad$ which leads to $\delta \dot{p}(t)=\tilde{H}_{I}^{d}\left(\delta \dot{\tilde{b}}(t)+\dot{u}_{1 d}(t)\right)+\tilde{H}_{P}^{d}\left(\delta \ddot{\tilde{b}}(t)+\dot{u}_{2 d}(t)\right)$.

Up to now, we studied what is a state-feedback control structure for the given dynamics and how to compensate for delays explicitly for continuous-time systems. We would like to note that any kinds of delays are easy to deal with for discrete-time systems. The natural next question is how to obtain a stabilizing gain of the feedback control structure. As a trial to compare impact of different AQM structures on performance, the present paper applies optimal control framework that allows us to measure deviation of the transients of state and control variables from the equilibrium.

\section{Stabilizing Gain Design and Its IMPACT ON PERFORMANCE}

In this section, we show how to get stabilizing optimal gains of the feedback control structures for the linearized systems (18) and (20) which can easily be applied to the cases of additional dynamics such as a low-pass filter and a modified virtual queue dynamics (see our companion papers [4] and [5] for the cases of additional dynamics). Then, the impact of each structure on performance is discussed from the results of the optimal control framework.

\section{A. A Stabilizing Optimal Gain for PD-Type Feedback Control}

As a performance measure for (18), we consider the following optimization problem:

$$
\min _{\delta p(\cdot)} J(s(t), \delta p(\cdot))
$$

with $J(s(t), \delta p(\cdot))=\int_{t}^{t+\infty}\left(s^{T}(\sigma) Q s(\sigma)+\delta p^{T}(\sigma)\right.$ $\left.R_{c} \delta p(\sigma)\right) d \sigma$, where the state weighting matrix $Q$ is nonnegative, the control weighting matrix $R_{c}$ is positive and the pair $\left(A, Q^{1 / 2}\right)$ is observable. By solving the above optimization problem, we can get stabilizing optimal and inverse optimal gains of the PD-type control.

Even if $Q$ is negative, we can get a stabilizing control if the system is stabilizable. However, we do not consider the detailed case. Without loss of generosity, the current paper sets the weighting matrices $Q$ as $Q=\operatorname{diag}\left(Q_{i}\right)$, where $Q_{1}>0$ and $Q_{2} \geq 0$.

For ease of explanation of the previous performance index, assume that $\tau^{*}=0$ (i.e., $s(t)=z(t)$ and $\hat{B}=B$ ). Then, we define the stabilizing optimal gain design problem as choosing a feedback control $\delta p(t)$ that minimizes the cost of transient around an equilibrium

$$
\min _{\delta p(\cdot)} J(z(t), \delta p(\cdot))
$$

with $J(z(t), \delta p(\cdot))=\int_{t}^{t+\infty}\left(Q_{1} \delta b^{2}(\sigma)+Q_{2} \delta \dot{b}^{2}(\sigma)+\right.$ $\left.\delta p^{2}(\sigma)\right) d \sigma$.

Each term in the integrand penalizes transients on the queue length, queue length rate and the fluctuation of the loss probability, respectively. Hence, the cost is a weighted sum of the transients weighted by $Q_{1}, Q_{2}$ and 1, respectively. For the given weighting, a slower transient incurs a higher cost.

Throughout the rest of this subsection, for simplicity of notation, we define

$$
\begin{aligned}
F_{1} & =A_{1}^{2}+\hat{B}_{1}^{2} Q_{1} \quad F_{2}=A_{2}^{2}+\hat{B}_{1}^{2} Q_{2} \\
a_{3} & =\frac{1}{\left(a_{1}-a_{2}\right)} \log _{e} \frac{a_{2}}{a_{1}} .
\end{aligned}
$$

Proposition 1: The stabilizing optimal gain of the PD-type delay-dependent control (19), which minimizes the transient cost (26) for system (18), is given by $H_{P}^{\tau}=\left(A_{1} e_{3}-e_{2} \sqrt{F_{1}}+\right.$ $\left.A_{1} e_{1} \sqrt{F_{2}+2 A_{1}+2 \sqrt{F_{1}}}\right) /\left(a_{1}-a_{2}\right) B_{1}$ and $H_{D}^{\tau}=\left(e_{1}\left(A_{1}+\right.\right.$ $\left.\left.\sqrt{F_{1}}\right)+e_{3}\left(A_{2}+\sqrt{F_{2}+2 A_{1}+2 \sqrt{F_{1}}}\right)\right) /\left(a_{1}-a_{2}\right) B_{1}$ and the resulting optimal cost is given by $J^{*}=s^{T}(0) K s(0)$, where $K$ satisfies $0=A^{T} K+K A+Q-K \hat{B} \hat{B}^{T} K$ (Refer to [1] and [3] for the solution of this algebraic Riccati equation).

When $\tau^{*}=0$ in (7), the stabilizing optimal gain of (9) is given by $H_{P}=-\left(A_{1}+\sqrt{A_{1}^{2}+B_{1}^{2} Q_{1}}\right) / B_{1}, H_{D}=-\left(A_{2}+\right.$ $\sqrt{\left.A_{2}^{2}+B_{1}^{2} Q_{2}+2 A_{1}+2 \sqrt{A_{1}^{2}+B_{1}^{2} Q_{1}}\right)} / B_{1}$.

If the state and input constraints are not violated, then $s_{1}(t)$ $\left(\delta b(t)\right.$ when $\left.\tau^{*}=0\right)$ is given by

$$
\begin{aligned}
s_{1}(t)= & \frac{1}{\left(\lambda_{2}-\lambda_{1}\right)}\left[\left(\lambda_{2} s_{1}(0)-s_{2}(0)\right) e^{\lambda_{1} t}\right. \\
& \left.\quad-\left(\lambda_{1} s_{1}(0)-s_{2}(0)\right) e^{\lambda_{2} t}\right] \text { when } \lambda_{1} \neq \lambda_{2} \\
= & {\left[s_{1}(0)+t\left(s_{2}(0)-s_{1}(0) \lambda_{1}\right)\right] e^{\lambda_{1} t} \text { when } \lambda_{1}=\lambda_{2}(27) } \\
\lambda_{1}, \lambda_{2}= & \frac{-\sqrt{F_{2}+2\left(A_{1}+\sqrt{F_{1}}\right)} \pm \sqrt{F_{2}+2\left(A_{1}-\sqrt{F_{1}}\right)}}{2} .
\end{aligned}
$$


Proof: The optimal control that minimizes (26) and the resulting optimal cost are given by

$$
\delta p^{*}(t)=-\hat{B}^{T} K s(t) \quad J^{*}(s(t))=s^{T}(t) K s(t) .
$$

Note that $K$ is a symmetric positive-definite matrix and the resulting closed-loop system is asymptotically stable since the pairs $(A, \hat{B})$ and $\left(A, Q^{1 / 2}\right)$ are controllable and observable, respectively [47]. By solving (29), we get the optimal gain. From the closed-loop system, we get (27) and (28).

Proposition 1 implies that the solution of problem (26) is a stabilizing feedback control, specified by $\left(H_{P}^{\tau}, H_{D}^{\tau}\right)$. Conversely, given any AQM of this structure, it solves problem (26) with appropriate weights $Q_{i}$ as the next result says. It can be easily proved from Proposition 1.

Proposition 2: Given a stabilizing control $\delta p(t)=$ $\left[H_{1}, H_{2}\right] s(t)$ that satisfies $A_{1}+\hat{B}_{1} H_{1}<0$ and $A_{2}+\hat{B}_{1} H_{2}<0$, it solves problem (26) with weights: $Q_{1}=\left(H_{1}^{2} \hat{B}_{1}+\right.$ $\left.2 H_{1} A_{1}\right) / \hat{B}_{1}, Q_{2}=\left(H_{2}^{2} \hat{B}_{1}+2 H_{2} A_{2}+2 H_{1}\right) / \hat{B}_{1}$. The corresponding closed-loop eigenvalues $\lambda_{1}$ and $\lambda_{2}$ are given by

$$
\lambda_{1}, \lambda_{2}=\frac{\left(A_{2}+\hat{B}_{1} H_{2}\right) \pm \sqrt{\left(A_{2}+\hat{B}_{1} H_{2}\right)^{2}+4\left(A_{1}+\hat{B}_{1} H_{1}\right)}}{2} .
$$

Proposition 3: Given eigenvalues $\lambda_{1}$ and $\lambda_{2}$ of the closedloop system (18), where real parts of $\lambda_{1}$ and $\lambda_{2}$ are negative, it solves problem (26) with weights: $Q_{1}=\left(\lambda_{1}^{2} \lambda_{2}^{2}-A_{1}^{2}\right) / \hat{B}_{1}^{2}$, $Q_{2}=\left(\lambda_{1}^{2}+\lambda_{2}^{2}-A_{2}^{2}-2 A_{1}\right) / \hat{B}_{1}^{2}$.

From Proposition 3, an easy way to design $Q_{1}$ and $Q_{2}$ is to make $\lambda_{1}$ equal to $\lambda_{2}$, i.e., $\lambda_{1}=\lambda_{2}$. It can be done by setting $Q_{1}=\left(\lambda^{4}-A_{1}^{2}\right) / \hat{B}_{1}^{2}, Q_{2}=\left(2 \sqrt{A_{1}^{2}+\hat{B}_{1}^{2} Q_{1}}-A_{2}^{2}-2 A_{1}\right) / \hat{B}_{1}^{2}$. Then, we have only to design one parameter for the secondorder system. As a loss probability, $\delta p(t)$ can be constrained as $-p^{*} \leq \delta p(t) \leq 1-p^{*}$. Thus, it is necessary to check the extremum of $\delta p(t)$. Refer to [1] for more details of this issue.

Similarly, we show how to get a stabilizing optimal gain of the PID-type control for the linearized system (20) in the next section.

\section{B. A Stabilizing Optimal Gain for PID-Type Feedback Control}

As a performance measure for (20), we consider

$$
\min _{\delta \dot{p}(\cdot)} J\left(s_{e}(t), \delta \dot{p}(\cdot)\right)
$$

with $J=\int_{t}^{t+\infty}\left(s_{e}^{T}(\sigma) Q s_{e}(\sigma)+\delta \dot{p}^{T}(\sigma) R_{c} \delta \dot{p}(\sigma)\right) d \sigma$, where $Q=Q^{T} \geq 0$ and the pair $\left(A_{e}, Q^{1 / 2}\right)$ is observable.

Without loss of generosity, we define $K_{e}=\left(K_{i j}\right)$ and $Q=$ $\operatorname{diag}\left(Q_{i}\right)$, where $K_{i j}$ is the $i$ th low and $j$ th column element and $Q_{i} \mathrm{~s}$ are nonnegative. For simplicity, we also define

$\hat{\lambda}_{1}=\lambda_{1}+\lambda_{2}+\lambda_{3}, \hat{\lambda}_{2}=\lambda_{1} \lambda_{2}+\lambda_{2} \lambda_{3}+\lambda_{1} \lambda_{3}, \hat{\lambda}_{3}=\lambda_{1} \lambda_{2} \lambda_{3}$.

Proposition 4: The stabilizing optimal gain of the PID-type delay-dependent control (21), which minimizes transient cost (30) for system (20), is given by $H_{I}^{\tau}=-\hat{B}_{1} K_{13}\left(\left(a_{2}-\right.\right.$ $\left.\left.a_{1}\right) e^{-A_{2} \tau^{*}} / e_{1}\right), H_{P}^{\tau}=-\hat{B}_{1}\left(K_{13} e_{4}-K_{23}\left(e_{2} / e_{1}\right)+K_{33} A_{1}\right)$,
$H_{D}^{\tau}=-\hat{B}_{1}\left(K_{13} e_{5}+K_{23}+K_{33}\left(e_{3} / e_{1}\right)\right)$ and the resulting optimal cost is given by

$$
J^{*}\left(s_{e}(0)\right)=s_{e}^{T}(0) K_{e} s_{e}(0)
$$

where $K_{e}$ is a symmetric positive-definite matrix satisfying $0=$ $A_{e}^{T} K_{e}+K_{e} A_{e}+Q-K_{e} \hat{B}_{e} \hat{B}_{e}^{T} K_{e}$. Refer to [1] and [3] for the detailed values of $K_{i j}$ for $i, j=1,2,3$ and $s_{1}(t)(\delta b(t)$ when $\tau^{*}=0$ ).

The proof of the previous proposition follows that of Proposition 1.

Proposition 4 implies that the solution of problem (30) is a feedback control algorithm, specified by $\left(K_{13}, K_{23}, K_{33}\right)$. Conversely, given any AQM of this structure, it solves problem (30) with appropriate weights $Q_{i}$, as the next result states.

Proposition 5: Given a stabilizing control $\delta \dot{p}(t)=$ $\left[\begin{array}{lll}H_{1} & H_{2} & H_{3}\end{array}\right] s_{e}(t)$, it solves problem (30) with weights: $Q_{1}=H_{1}^{2}, Q_{2}=H_{2}^{2}-2\left(\left(A_{2} H_{1}+\hat{B}_{1} H_{1} H_{3}-A_{1} H_{2}\right) / \hat{B}_{1}\right)$, $Q_{3}=H_{3}^{2}+2\left(\left(A_{2} H_{3}+H_{2}\right) / \hat{B}_{1}\right)$. Then, $K_{11}, K_{12}, K_{22}$, $K_{13}, K_{23}$, and $K_{33}$ are given by $K_{11}=\left(A_{1}+\hat{B}_{1} H_{2}\right) H_{1} / \hat{B}_{1}$, $K_{12}=\left(A_{2}+\hat{B}_{1} H_{3}\right) H_{1} / \hat{B}_{1}, K_{22}=H_{2} H_{3}+\left(\left(A_{1} H_{3}+\right.\right.$ $\left.\left.A_{2} H_{2}+H_{1}\right) / \hat{B}_{1}\right), K_{13}=-\left(H_{1} / \hat{B}_{1}\right), K_{23}=-\left(H_{2} / \hat{B}_{1}\right)$, $K_{33}=-\left(H_{3} / \hat{B}_{1}\right)$, and $\hat{\lambda}_{1}, \hat{\lambda}_{2}, \hat{\lambda}_{3}$ are given by

$$
\hat{\lambda}_{1}=A_{2}+\hat{B}_{1} H_{3} \quad \hat{\lambda}_{2}=-\left(A_{1}+\hat{B}_{1} H_{2}\right) \quad \hat{\lambda}_{3}=\hat{B}_{1} H_{1} .
$$

Proposition 6: Given eigenvalues $\lambda_{1}, \lambda_{2}$, and $\lambda_{3}$ of the closed-loop system (20), where real parts of $\lambda_{1}, \lambda_{2}$, and $\lambda_{3}$ are negative, $\delta \dot{p}(t)$ solves problem (30) with weights $Q_{1}=\hat{\lambda}_{3}^{2} / \hat{B}_{1}^{2}, Q_{2}=\left(-A_{1}^{2}+\hat{\lambda}_{2}^{2}-2 \hat{\lambda}_{1} \hat{\lambda}_{3}\right) / \hat{B}_{1}^{2}, Q_{3}=$ $\left(-A_{2}^{2}-2 A_{1}+\hat{\lambda}_{1}^{2}-2 \hat{\lambda}_{2}\right) / \hat{B}_{1}^{2}$. Then, $K_{13}, K_{23}, K_{33}, K_{11}, K_{12}$, and $K_{22}$ are given by $K_{11}=-\left(\hat{\lambda}_{2} \hat{\lambda}_{3} / \hat{B}_{1}^{2}\right), K_{12}=\hat{\lambda}_{1} \hat{\lambda}_{3} / \hat{B}_{1}^{2}$, $K_{22}=\left(-A_{1} A_{2}-\hat{\lambda}_{1} \hat{\lambda}_{2}+\hat{\lambda}_{3}\right) / \hat{B}_{1}^{2}, K_{13}=-\left(\hat{\lambda}_{3} / \hat{B}_{1}^{2}\right)$, $K_{23}=\left(A_{1}+\hat{\lambda}_{2}\right) / \hat{B}_{1}^{2}, K_{33}=\left(A_{2}-\hat{\lambda}_{1}\right) / \hat{B}_{1}^{2}$.

Similarly to the previous section, an easy way to design $Q_{1}$, $Q_{2}$, and $Q_{3}$ is to make $\lambda_{1}, \lambda_{2}$, and $\lambda_{3}$ equal, i.e., $\lambda_{1}=\lambda_{2}=\lambda_{3}$. It can be done by setting $Q_{1}=\left(\lambda^{3} / \hat{B}_{1}\right)^{2}, Q_{2}=\left(-A_{1}^{2}+\right.$ $\left.3 \lambda^{4}\right) / \hat{B}_{1}^{2}, Q_{3}=\left(-A_{2}^{2}-2 A_{1}+3 \lambda^{2}\right) / \hat{B}_{1}^{2}$. Then, we have only to design one parameter for the third-order system.

We now interpret the impact of each structure on performance from the results of the PID-type optimal control framework. The current paper mainly focus on AQM algorithms such as RED, REM, and PI based on the real queue dynamics. For a brief discussion about AVQ based on the virtual queue dynamics, see Section IV-B.

\section{Impact of Different AQM Structures on Performance}

For ease of comparison, we assume that $\tau^{*}=0$ (i.e., $s_{e}(t)=$ $z_{e}(t), \hat{B}_{e}=B_{e}$ ) for the linearized model and we do not consider the low-pass filter of RED (For more details, see [4]).

Then, the linear models of RED and REM/PI are

$$
\begin{aligned}
\text { simplified RED : } & \delta \dot{p}^{r}(t)=H_{2}^{r} \delta \dot{b}(t) \\
\mathrm{REM} / \mathrm{PI}: & \delta \dot{p}^{m}(t)=H_{1}^{m} \delta b(t)+H_{2}^{m} \delta \dot{b}(t)
\end{aligned}
$$

for some nonnegative constants $H_{2}^{r}, H_{1}^{m}, H_{2}^{m}$. The linear models of RED and REM/PI roughly capture the models in [10], [16], and [19]. 
By Proposition 4, the stabilizing optimal AQM has a strictly positive gain $K_{33}>0$. Since this condition is satisfied by none of RED, REM and PI, none of them can be made optimal in the sense of minimizing (30). We can also interpret their structural deficiency of D-type control as follows.

From (32) in Proposition 5, the sum of eigenvalues of the closed-loop system with REM and PI is given by $\hat{\lambda}_{1}=A_{2}$, while our PID-type AQM has $\hat{\lambda}_{1}=A_{2}+B_{1} H_{3}$. Thus, we cannot adjust the sum of eigenvalues without D-type control structure, i.e., cannot control the dynamic behavior of the closed-loop system arbitrary while we can do that with the state-feedback control structures. For example, $\hat{\lambda}_{1}$ is less negative when $H_{3}=0$ than when $H_{3}>0$. This suggests that the decaying rate is smaller with $H_{3}=0$. We can similarly interpret the structural deficiency of P-type RED, compared with the PD-type state-feedback AQM from Propositions 1 and 2.

As shown in (31), transient costs of a simplified RED and REM/PI can be obtained from (31) by setting some elements of $K$ to zero, with $H_{1}=H_{3}=0$ (i.e., $K_{11}=K_{12}=K_{13}=$ $K_{33}=0$ ) and with $H_{3}=0$ (i.e., $K_{33}=0$ ), respectively. Note that the costs of RED and REM/PI are always greater than that of the stabilizing optimal AQM since (31) is the optimal cost for the given system and weighting matrices.

Until now, we considered single link and homogeneous sources. In the next section, we come back to the general networks with multiple links and heterogeneous delays of Section II-B.

\section{EXTENSION TO MULTIPLE LINKS AND HETEROGENEOUS SOURCES}

In this section, we extend the results in the previous sections to the case of multiple links and heterogeneous sources. First, we derive an equivalent nonlinear system and a linearized statespace model to this general case. Then, delay compensation and stabilizing optimal gain design are studied.

\section{A. State-Space Model for General Networks}

To derive a state-space model for the general case, we define

$$
\begin{aligned}
w(t) & =\left[\begin{array}{c}
w_{1}(t) \\
w_{2}(t) \\
\vdots \\
w_{N}(t)
\end{array}\right] \quad b(t)=\left[\begin{array}{c}
b_{1}(t) \\
b_{2}(t) \\
\vdots \\
b_{L}(t)
\end{array}\right] \quad p(t)=\left[\begin{array}{c}
p_{1}(t) \\
p_{2}(t) \\
\vdots \\
p_{L}(t)
\end{array}\right] \\
R_{s} & =\left[\begin{array}{ll}
\left.R_{1 s}^{T}, R_{2 s}^{T}, \ldots R_{L s}^{T}\right]^{T} \quad R_{l}=\left[R_{l 1}, R_{l 2}, \ldots, R_{l N}\right.
\end{array}\right] \\
D_{1} & =\operatorname{diag}\left(\frac{1}{c_{l}}\right) \quad D_{2}=\operatorname{diag}\left(\frac{1}{\tau_{s}^{*}}\right) \\
A_{1 l} & =-R_{l} \operatorname{diag}\left(\frac{2 w_{s}^{* 2}}{\tau_{s}^{* 3}\left(2+w_{s}^{* 2}\right)} R_{s}^{T} D_{1}\right) \\
A_{2 l} & =-R_{l} \operatorname{diag}\left(\frac{2 w_{s}^{*}}{\tau_{s}^{*}\left(2+w_{s}^{* 2}\right)}\right) R_{l}^{-1} \\
A_{3 l} & =-R_{l} \operatorname{diag}\left(\frac{w_{s}^{*}}{\tau_{s}^{* 2}} R_{s}^{T} D_{1}\right) \\
B_{l} & =-R_{l} \operatorname{diag}\left(\frac{\left(2+w_{s}^{* 2}\right)}{2 \tau_{s}^{* 2}} R_{s}^{T}\right), \alpha_{l s}=\operatorname{diag}\left(A_{1 l}\right) E_{l}^{1} E_{s}^{2} \\
\beta_{l s} & =\operatorname{diag}\left(A_{3 l}\right) E_{l}^{1} E_{s}^{2} \gamma_{l s}=\operatorname{diag}\left(B_{l}\right) E_{l}^{1} E_{s}^{2}
\end{aligned}
$$

where $R_{l}^{-1}$ is a vector satisfying $R_{l} R_{l}^{-1}=1$, $E_{l}^{1} \in \mathbb{R}^{(L \times L \times N) \times(L \times N)}$ and $E_{s}^{2} \in \mathbb{R}^{(N \times L) \times L}$ have identity matrices $I_{l} \in \mathbb{R}^{(L \times N) \times(L \times N)}$ and $I_{s} \in \mathbb{R}^{L \times L}$ only at $l$ th and $s$ th block, respectively, and zero matrices at the other blocks. As an exceptional definition, when $\tau_{s}^{b *}$ is used inside the feedback control variable $p(\cdot)$ like $p\left(-\tau_{s}^{b *}\right)$ and $p\left(t-\tau_{l s}^{f *}-\tau_{s}^{b *}\right)$, we mean that $p\left(-\tau_{s}^{b *}\right)=\left[p_{1}\left(-\tau_{1 s}^{b *}\right), p_{2}\left(-\tau_{2 s}^{b *}\right), \ldots, p_{L}\left(-\tau_{L s}^{b *}\right)\right]^{T}$ and $p\left(t-\tau_{l s}^{f *}-\tau_{s}^{b *}\right)=\left[p_{1}\left(t-\tau_{l_{s}}^{f *}-\right.\right.$ $\left.\left.\tau_{1 s}^{b *}\right), p_{2}\left(t-\tau_{l s}^{f *}-\tau_{2 s}^{b *}\right), \ldots, p_{L}\left(t-\tau_{l s}^{f *}-\tau_{L s}^{b *}\right)\right]^{T}$

Differentiating (2) and rearranging the differentiated equation with (1) and (2), we have

$$
\begin{aligned}
& \ddot{b}_{l}(t)=\sum_{s} R_{l s}\left[\frac{\left(1-\sum_{k} R_{k s} p_{k}\left(t-\tau_{l s}^{f *}-\tau_{k s}^{b *}\right)\right)}{\tau_{s}\left(t-\tau_{l s}^{f *}\right) \tau_{s}\left(t-\tau_{l s}^{f *}-\tau_{s}^{*}\right)}\right. \\
& \times \frac{w_{s}\left(t-\tau_{l s}^{f *}-\tau_{s}^{*}\right)}{w_{s}\left(t-\tau_{l s}^{f *}\right)} \\
&-\frac{1}{2} \frac{w_{s}\left(t-\tau_{l s}^{f *}-\tau_{s}^{*}\right)}{\tau_{s}\left(t-\tau_{l s}^{f *}-\tau_{s}^{*}\right)} \frac{w_{s}\left(t-\tau_{l s}^{f *}\right)}{\tau_{s}\left(t-\tau_{l s}^{f *}\right)} \\
& \times\left(\sum_{k} R_{k s} p_{k}\left(t-\tau_{l s}^{f *}-\tau_{k s}^{b *}\right)\right) \\
&-\frac{w_{s}\left(t-\tau_{l s}^{f *}\right)}{\tau_{s}^{2}\left(t-\tau_{l s}^{f *}\right)} \\
&\left.\times\left(\sum_{k} R_{k s} \frac{\dot{b}_{k}\left(t-\tau_{l s}^{f *}\right)}{c_{k}}\right)\right] .
\end{aligned}
$$

From (33), we can get the following linearized TCP model of the general case:

$$
\begin{array}{r}
\dot{z}(t)=\left[\begin{array}{cc}
0 & I \\
0 & \operatorname{diag}\left(A_{2 l}\right)
\end{array}\right] z(t)+\sum_{s} \sum_{l}\left[\begin{array}{cc}
0 & 0 \\
\alpha_{l s} & \beta_{l s}
\end{array}\right] z\left(t-\tau_{l s}^{f *}\right) \\
+\sum_{s} \sum_{l}\left[\begin{array}{c}
0 \\
\gamma_{l s}
\end{array}\right] \delta p\left(t-\tau_{l s}^{f *}-\tau_{s}^{b *}\right)
\end{array}
$$

where $\left\{z(\sigma), \sigma \in\left[-\tau_{l s}^{f *}, 0\right]\right\}$ and $\left\{\delta p(\sigma), \sigma \in\left[-\tau_{l s}^{f *}-\tau_{s}^{b *}, 0\right]\right\}$ are given, and $z(t)=\left[\begin{array}{l}\delta b(t) \\ \delta \dot{b}(t)\end{array}\right]$.

If we want integral control action, we have another differentiation given by

$$
\begin{aligned}
\dddot{b}_{l}(t)=\sum_{s} g_{s}( & w_{s}\left(t-\tau_{l s}^{f *}-\tau_{s}^{*}\right), \dot{w}_{s}\left(t-\tau_{l s}^{f *}-\tau_{s}^{*}\right), \\
& w_{s}\left(t-\tau_{l s}^{f *}\right), \dot{w}_{s}\left(t-\tau_{l s}^{f *}\right), b\left(t-\tau_{l s}^{f *}\right), \\
& \dot{b}\left(t-\tau_{l s}^{f *}\right), \ddot{b}\left(t-\tau_{l s}^{f *}\right), b\left(t-\tau_{l s}^{f *}-\tau_{s}^{*}\right), \\
& \dot{b}\left(t-\tau_{l s}^{f *}-\tau_{s}^{*}\right), p\left(t-\tau_{l s}^{f *}-\tau_{s}^{b *}\right), \\
& \left.\dot{p}\left(t-\tau_{l s}^{f *}-\tau_{s}^{b *}\right)\right) .
\end{aligned}
$$


From (35), we can get the extended linearized AIMD model of the general case

$$
\begin{aligned}
\dot{z}_{e}(t)=A_{e} z_{e}(t)+\sum_{s} & \sum_{l} A_{l s} z_{e}\left(t-\tau_{l s}^{f *}\right) \\
& +\sum_{s} \sum_{l} B_{l s} \delta \dot{p}\left(t-\tau_{l s}^{f *}-\tau_{s}^{b *}\right)
\end{aligned}
$$

where $\left\{z_{e}(\sigma), \sigma \in\left[-\tau_{l s}^{f *}, 0\right]\right\}$ and $\left\{\delta \dot{p}(\sigma), \sigma \in\left[-\tau_{l s}^{f *}-\right.\right.$ $\left.\left.\tau_{s}^{b *}, 0\right]\right\}$ are given, and

$$
\begin{gathered}
z_{e}(t)=\left[\begin{array}{c}
\delta b(t) \\
\delta \dot{b}(t) \\
\delta \ddot{b}(t)
\end{array}\right] \quad A_{e}=\left[\begin{array}{ccc}
0 & I & 0 \\
0 & 0 & I \\
0 & 0 & \operatorname{diag}\left(A_{2 l}\right)
\end{array}\right] \\
A_{l s}=\left[\begin{array}{ccc}
0 & 0 & 0 \\
0 & 0 & 0 \\
0 & \alpha_{l s} & \beta_{l s}
\end{array}\right] \quad B_{l s}=\left[\begin{array}{c}
0 \\
0 \\
\gamma_{l s}
\end{array}\right] .
\end{gathered}
$$

For detailed derivation of (34) and (36), refer to Appendix III. As shown in the previous equations, the state-feedback controls of (34) and (36) also have PD-type and PID-type state-feedback control structures as in (9) and (17) if we ignore all delays.

\section{B. Delay Compensation for General Networks}

In the same way as Section IV, we first consider how to compensate for delays in the feedback control. If $\tau_{l s}^{f *}=0$ for all $l$ and $s,(36)$ can be converted to the equivalent system

$$
\dot{z}_{e}(t)=A_{e} z_{e}(t)+\sum_{s} B_{s} \delta \dot{p}\left(t-\tau_{s}^{b *}\right)
$$

where $z_{e}(0)$ and $\left\{\delta \dot{p}(\sigma), \sigma \in\left[-\tau_{s}^{b *}, 0\right]\right\}$ are given

$$
A_{e}=\left[\begin{array}{ccc}
0 & I & 0 \\
0 & 0 & I \\
0 & A_{1} & A_{2}
\end{array}\right] \quad B_{s}=\left[\begin{array}{c}
0 \\
0 \\
\eta_{s}
\end{array}\right]
$$

$A_{1}=-R \operatorname{diag}\left(2 w_{s}^{* 2} / \tau_{s}^{* 3}\left(2+w_{s}^{* 2}\right)\right) R^{T} D_{1}, A_{2}=\operatorname{diag}\left(A_{2 l}\right)-$ $R \operatorname{diag}\left(w_{s}^{*} / \tau_{s}^{* 2}\right) R^{T} D_{1}$

$$
\eta_{s}=\left[\begin{array}{c}
-R_{1 s}\left(\left(2+w_{s}^{* 2}\right) / 2 \tau_{s}^{* 2}\right) R_{s}^{T} \\
\vdots \\
-R_{L s}\left(\left(2+w_{s}^{* 2}\right) / 2 \tau_{s}^{* 2}\right) R_{s}^{T}
\end{array}\right] .
$$

Define

$$
h_{e}(t)=z_{e}(t)+\sum_{s} \sum_{k} \int_{t}^{t+\tau_{k s}^{b *}} e^{A_{e}(t-\sigma)}\left[\begin{array}{c}
0 \\
0 \\
\eta_{s} E_{k}
\end{array}\right]
$$

$\delta \dot{p}_{k}\left(\sigma-\tau_{k s}^{b *}\right) d \sigma$, where $E_{k}$ is a $L$-column vector that has 1 in $l$ th row and 0 in other rows. Then, the delayed system (37) can be converted to the equivalent nominal system

$$
\begin{aligned}
\dot{h}_{e}(t)=A_{e} h_{e}(t)+\sum_{s} & {\left[\Phi_{A_{e}}\left(t, t+\tau_{1 s}^{b *}\right)\left[\begin{array}{c}
0 \\
0 \\
\eta_{s} E_{1}
\end{array}\right], \ldots,\right.} \\
& \left.\Phi_{A_{e}}\left(t, t+\tau_{L s}^{b *}\right)\left[\begin{array}{c}
0 \\
0 \\
\eta_{s} E_{L}
\end{array}\right]\right] \delta \dot{p}(t)
\end{aligned}
$$$$
=A_{e} h_{e}(t)+\tilde{B}_{e} \delta \dot{p}(t) .
$$

To get a more simplified equivalent system, we have one more transformation. Let $s_{e}(t)=T_{e} h_{e}(t)$ and $\tilde{B}_{e}=\left[\begin{array}{c}\tilde{B}_{1} \\ \tilde{B}_{2} \\ \tilde{B}_{3}\end{array}\right]$, where
$T_{e}=\left[\begin{array}{ccc}-\tilde{B}_{3} \tilde{B}_{2}^{-1}-e_{6} A_{1} & I-e_{6} A_{2} & e_{6} \\ 0 & -\tilde{B}_{3} \tilde{B}_{2}^{-1} & I \\ 0 & A_{1} & A_{2}-\tilde{B}_{3} \tilde{B}_{2}^{-1}\end{array}\right]$ $e_{6}=\left(\tilde{B}_{2}-\tilde{B}_{3} \tilde{B}_{2}^{-1} \tilde{B}_{1}\right)\left(A_{1} \tilde{B}_{1}+A_{2} \tilde{B}_{2}-\tilde{B}_{3}\right)^{-1}, \tilde{B}_{3} \tilde{B}_{2}^{-1}+$ $e_{6} A_{1} \neq 0$, and $A_{1}+\left(A_{2}-\tilde{B}_{3} \tilde{B}_{2}^{-1}\right) \tilde{B}_{3} \tilde{B}_{2}^{-1} \neq 0$.

Then, (38) can be rewritten as

$$
\dot{s}_{e}(t)=\hat{A}_{e} s_{e}(t)+\hat{B}_{e} \delta \dot{p}(t)
$$

where

$$
\hat{A}_{e}=\left[\begin{array}{ccc}
0 & I & 0 \\
0 & 0 & I \\
0 & \hat{A}_{1} & \hat{A}_{2}
\end{array}\right] \quad \hat{B}_{e}=\left[\begin{array}{c}
0 \\
0 \\
\hat{B}_{1}
\end{array}\right]
$$

$\hat{A}_{1}=\hat{B}_{1} e_{7} A_{1} \tilde{B}_{2} \tilde{B}_{3}^{-1}, \hat{A}_{2}=A_{2}-\tilde{B}_{3} \tilde{B}_{2}^{-1}+\hat{B}_{1} e_{7}, \hat{B}_{1}=$ $A_{1} \tilde{B}_{2}+A_{2} \tilde{B}_{3}-\tilde{B}_{3} \tilde{B}_{2}^{-1} \tilde{B}_{3}, e_{7}=\left(A_{2} \tilde{B}_{2}+A_{1} \tilde{B}_{2} \tilde{B}_{3}^{-1} \tilde{B}_{2}-\right.$ $\left.\tilde{B}_{3}\right)^{-1}$.

If $\tau_{l s}^{b *}=0$ for all $l$ and $s$, then $s_{e}(t)=z_{e}(t)$ and $\hat{B}_{1}=$ $-R \operatorname{diag}\left(\left(2+w_{s}^{* 2}\right) / 2 \tau_{s}^{* 2}\right) R^{T}$.

From this state-space model, we can get a PID-type delaydependent state-feedback control as in (21) (in the same way, we can get a PD-type delay-dependent state-feedback control). It is also easy to see that we can relax the assumption $\tau_{l s}^{f *}=0$ using the virtual queue dynamics as in Section IV-B.

\section{A Stabilizing Optimal Gain Design for General Networks}

In the same way as those of Propositions 4 and 5, we can get the stabilizing optimal gain solving (30) and consider the inverse optimal control under the assumption that the system is controllable and observable (refer to [2] for the details).

From the study in this section, we can see that the design procedures and structural properties for the case of single link and homogeneous sources hold for the general network case.

\section{CONCLUSION}

This paper studied how to design a stabilizing feedback control based on the state-space approach for the given TCP and its variants with additional dynamics, where the feedback control can be implemented either at sources or at routers with a different type of congestion signal. We derived state-feedback control and explicit delay compensation structures which are necessary to regulate the given dynamical system arbitrarily. As a subsequent result, we proposed a mathematical framework allowing us to interpret RED and REM/PI as different approximations of the unified framework, and discussed the impact of each structure on performance from the results of the stabilizing optimal control design.

One can extend this work to more realistic congestion control problems. We also expect that our feedback control design procedures can be applied to the future TCP protocol like the high-speed TCP [52]-[54] or other dynamical systems. 


\section{APPENDIX I}

DERIVATION OF (16)

Let $\left(w^{*}, b^{*}, p^{*}\right)$ be the equilibrium point. Then, the linearized model of (5) is

$$
\begin{aligned}
\delta \ddot{b}(t)= & \left.\frac{\partial f}{\partial b(t)}\right|_{*} \delta b(t)+\left.\frac{\partial f}{\partial \dot{b}(t)}\right|_{*} \delta \dot{b}(t) \\
& +\left.\frac{\partial f}{\partial \dot{b}\left(t-\tau^{*}\right)}\right|_{*} \delta \dot{b}\left(t-\tau^{*}\right)+\left.\frac{\partial f}{\partial p\left(t-\tau^{*}\right)}\right|_{*} \delta p\left(t-\tau^{*}\right)
\end{aligned}
$$

where $\partial f /\left.\partial b(t)\right|_{*}=-\left(2 N / \tau^{* 3} c\right)\left(1-p^{*}\right), \partial f /\left.\partial \dot{b}(t)\right|_{*}=$ $-\left(N / \tau^{* 2} c\right)\left(1-p^{*}\right)-(1 / \tau)-\left(p^{*} c / 2 N\right), \partial f /\left.\partial \dot{b}\left(t-\tau^{*}\right)\right|_{*}=0$, $\partial f /\left.\partial p\left(t-\tau^{*}\right)\right|_{*}=-\left(N / \tau^{* 2}\right)-\left(c^{2} / 2 N\right), p^{*}=2 N^{2} /\left(2 N^{2}+\right.$ $c^{2} \tau^{* 2}$ ). Then the linearized model of (5) can be converted to (6).

Similarly, the linearized model of (15) is

$$
\begin{aligned}
& \delta \dddot{b}^{\prime}(t)=\left.\frac{\partial g}{\partial b(t)}\right|_{*} \delta b(t)+\left.\frac{\partial g}{\partial \dot{b}(t)}\right|_{*} \delta \dot{b}(t)+\left.\frac{\partial g}{\partial \ddot{b}(t)}\right|_{*} \delta \ddot{b}(t) \\
& +\left.\frac{\partial g}{\partial \dot{b}\left(t-\tau^{*}\right)}\right|_{*} \delta \dot{b}\left(t-\tau^{*}\right) \\
& +\left.\frac{\partial g}{\partial \ddot{b}\left(t-\tau^{*}\right)}\right|_{*} \delta \ddot{b}\left(t-\tau^{*}\right) \\
& +\left.\frac{\partial g}{\partial p\left(t-\tau^{*}\right)}\right|_{*} \delta p\left(t-\tau^{*}\right) \\
& +\left.\frac{\partial g}{\partial \dot{p}\left(t-\tau^{*}\right)}\right|_{*} \delta \dot{p}\left(t-\tau^{*}\right) \\
& =\left.\frac{\partial f}{\partial b(t)}\right|_{*} \delta \dot{b}(t)+\left.\frac{\partial f}{\partial \dot{b}(t)}\right|_{*} \delta \ddot{b}(t) \\
& +\left.\frac{\partial f}{\partial p\left(t-\tau^{*}\right)}\right|_{*} \delta \dot{p}\left(t-\tau^{*}\right) .
\end{aligned}
$$

Then, the linearized model of (15) can be converted to (16).

\section{APPENDIX II}

\section{DERIVATION OF (18) AND (20)}

We have only to derive (20) since we can handle (18) as a special case of (20).

Note that (16) can be rewritten as

$z_{e}\left(t+\tau^{*}\right)=e^{A_{e} \tau^{*}}\left[z_{e}(t)+\int_{-\tau^{*}}^{0} e^{-A_{e}\left(\sigma+\tau^{*}\right)} B_{e} \delta \dot{p}(\sigma+t) d \sigma\right]$ where

$$
e^{A_{e} t}=\frac{1}{\left(a_{2}-a_{1}\right)}\left[\begin{array}{cc}
\left(a_{2}-a_{1}\right) & (1,2) \\
0 & a_{2} e^{a_{1} t}-a_{1} e^{a_{2} t} \\
0 & a_{1} a_{2}\left(e^{a_{1} t}-e^{a_{2} t}\right)
\end{array}\right.
$$

with $(1,2)=\left(\left(a_{1}^{2}-a_{2}^{2}\right) / a_{1} a_{2}\right)+\left(a_{2} / a_{1}\right) e^{a_{1} t}-\left(a_{1} / a_{2}\right) e^{a_{2} t}$, $(1,3)=\left(\left(a_{2}-a_{1}\right) / a_{1} a_{2}\right)-\left(1 / a_{1}\right) e^{a_{1} t}+\left(1 / a_{2}\right) e^{a_{2} t},(2,3)=$ $-e^{a_{1} t}+e^{a_{2} t}$ and $(3,3)=-a_{1} e^{a_{1} t}+a_{2} e^{a_{2} t}$.

Define the insider part of the previous second equation as

$$
\begin{aligned}
h_{e}(t) & =z_{e}(t)+\int_{-\tau^{*}}^{0} e^{-A_{e}\left(\sigma+\tau^{*}\right)} B_{e} \delta \dot{p}(t+\sigma) d \sigma \\
& =z_{e}(t)+\left[\begin{array}{c}
\dot{u}_{1 \tau}(t) \\
\dot{u}_{2 \tau}(t) \\
\dot{u}_{3 \tau}(t)
\end{array}\right] .
\end{aligned}
$$

Using (40) and (41), system (16) can be rewritten as the following nominal system:

$$
\dot{h}_{e}(t)=A_{e} h_{e}(t)+\tilde{B}_{e} \delta \dot{p}(t)
$$

where

$$
\tilde{B}_{e}=\left[\begin{array}{c}
\tilde{B}_{1} \\
\tilde{B}_{2} \\
\tilde{B}_{3}
\end{array}\right]=e^{-A_{e} \tau^{*}} B_{e}=\left(B_{1} /\left(a_{1}-a_{2}\right)\right)\left[\begin{array}{c}
(1,1) \\
(2,1) \\
(3,1)
\end{array}\right]
$$

with $(1,1)=\left(\left(a_{1}-a_{2}\right) / a_{1} a_{2}\right)+\left(1 / a_{1}\right) e^{-a_{1} \tau^{*}}-$ $\left(1 / a_{2}\right) e^{-a_{2} \tau^{*}},(2,1)=e^{-a_{1} \tau^{*}}-e^{-a_{2} \tau^{*}}$ and $(3,1)=$ $a_{1} e^{-a_{1} \tau^{*}}-a_{2} e^{-a_{2} \tau^{*}}$.

To get the explicit stabilizing optimal gain as shown in Propositions 1 and 4, we have another transformation as follows.

Let $s_{e}(t)=T_{e} h_{e}(t)$, where the equation shown at the bottom of the page holds. $T_{e}$ can be rewritten as

$$
T_{e}=\left[\begin{array}{ccc}
\left(\left(a_{2}-a_{1}\right) e^{-\left(a_{1}+a_{2}\right) \tau^{*}} / e_{1}\right) & e_{4} & e_{5} \\
0 & -\left(e_{2} / e_{1}\right) & 1 \\
0 & A_{1} & \left(e_{3} / e_{1}\right)
\end{array}\right]
$$

$$
T_{e}=\left[\begin{array}{ccc}
-\frac{\left(A_{1} \tilde{B}_{2}^{2}+A_{2} \tilde{B}_{2} \tilde{B}_{3}-\tilde{B}_{3}^{2}\right)}{\tilde{B}_{2}\left(A_{1} \tilde{B}_{1}+A_{2} \tilde{B}_{2}-\tilde{B}_{3}\right)} & \frac{\left(A_{1} \tilde{B}_{1} \tilde{B}_{2}+A_{2} \tilde{B}_{1} \tilde{B}_{3}-\tilde{B}_{2} \tilde{B}_{3}\right)}{\tilde{B}_{2}\left(A_{1} \tilde{B}_{1}+A_{2} \tilde{B}_{2}-\tilde{B}_{3}\right)} & \frac{\left(\tilde{B}_{2}^{2}-\tilde{B}_{1} \tilde{B}_{3}\right)}{\tilde{B}_{2}\left(A_{1} \tilde{B}_{1}+A_{2} \tilde{B}_{2}-\tilde{B}_{3}\right)} \\
0 & -\frac{\tilde{B}_{3}}{\tilde{B}_{2}} & 1 \\
0 & A_{1} & \frac{\left(A_{2} \tilde{B}_{2}-\tilde{B}_{3}\right)}{\tilde{B}_{2}}
\end{array}\right]
$$


using the following equations:

$$
\begin{aligned}
& \frac{\left(A_{1} \tilde{B}_{2}^{2}+A_{2} \tilde{B}_{2} \tilde{B}_{3}-\tilde{B}_{3}^{2}\right)}{\tilde{B}_{2}} \\
& =-\frac{B_{1}^{2}}{\tilde{B}_{2}} e^{-\left(a_{1}+a_{2}\right) \tau^{*}} \\
& \quad=\frac{B_{1}\left(a_{2}-a_{1}\right)}{e_{1}} e^{-\left(a_{1}+a_{2}\right) \tau^{*}} \\
& \left(A_{1} \tilde{B}_{1}+A_{2} \tilde{B}_{2}-\tilde{B}_{3}\right) \\
& =-B_{1} \frac{\left(A_{1} \tilde{B}_{1} \tilde{B}_{2}+A_{2} \tilde{B}_{1} \tilde{B}_{3}-\tilde{B}_{2} \tilde{B}_{3}\right)}{\tilde{B}_{2}} \\
& =\frac{B_{1}}{e_{1}}\left[\frac{a_{1}}{a_{2}} e^{-a_{1} \tau^{*}}-\frac{a_{2}}{a_{1}} e^{-a_{2} \tau^{*}}+\frac{\left(a_{2}^{2}-a_{1}^{2}\right)}{a_{1} a_{2}} e^{-\left(a_{1}+a_{2}\right) \tau^{*}}\right] \\
& \left(\tilde{B}_{2}^{2}-\tilde{B}_{1} \tilde{B}_{3}\right) \\
& \quad \tilde{B}_{2} \\
& =-\frac{\tilde{B}_{1}}{e_{1}}\left[\frac{1}{a_{2}} e^{-a_{1} \tau^{*}}-\frac{1}{a_{1}} e^{-a_{2} \tau^{*}}+\frac{\left(a_{2}-a_{1}\right)}{a_{1} a_{2}} e^{-\left(a_{1}+a_{2}\right) \tau^{*}}\right] .
\end{aligned}
$$

Since $a_{2}<a_{1}<0$ for system matrices of (16), we have $\tilde{B}_{1}<0, \tilde{B}_{2}>0$, and $\tilde{B}_{3}<0$ for $\tau^{*}>0$. Since $\operatorname{det}(S)=$ $\left(B_{1}^{3} / \tilde{B}_{2}^{3}\right) \neq 0$, there exists $S^{-1}$. Thus, using the transformation $s_{e}(t)=T_{e} h_{e}(t)$, we can rewrite system (42) as

$$
\begin{aligned}
\dot{s}_{e}(t)=S A_{e} S^{-1} s(t) & +S \tilde{B}_{e} \delta \dot{p}(t)=A_{e} s_{e}(t) \\
& +\left[\begin{array}{c}
0 \\
0 \\
\left(B_{1}\left(a_{2}-a_{1}\right) / e_{1}\right) e^{-\left(a_{1}+a_{2}\right) \tau^{*}}
\end{array}\right] \delta \dot{p}(t)
\end{aligned}
$$

Similarly, we get (18).

\section{APPENDIX III}

\section{DERIVATION OF (34) AND (36)}

We have only to show how to derive (36) since we can handle (34) as a special case of (36). Linearizing (35) and using $R_{s}^{T} p^{*}=\left(1 /\left(1+\left(w_{s}^{* 2} / 2\right)\right)\right)$ and $R_{l} D_{2} w^{*}=c_{l}$, we have $\left.\left(\partial g_{s} / \partial \dot{w}_{s}\left(t-\tau_{l s}^{f *}\right)\right)\right|_{*}=-R_{l_{s}}\left(2 w_{s}^{*} / \tau_{s}^{* 2}\left(2+w_{s}^{* 2}\right)\right)$, $\left.\left(\partial g_{s} / \partial \ddot{b}\left(t-\tau_{l s}^{f *}\right)\right)\right|_{*}=-R_{l s}\left(w_{s}^{*} / \tau_{s}^{* 2}\right) R_{s}^{T} D_{1},\left(\partial g_{s} / \partial \dot{p}(t-\right.$ $\left.\left.\tau_{l s}^{f *}-\tau_{s}^{b *}\right)\right)\left.\right|_{*}=-R_{l s}\left(\left(2+w_{s}^{* 2}\right) / 2 \tau_{s}^{* 2}\right) R_{s}^{T}$, where the other terms are zero.

Linearizing (33), we have

$\delta \ddot{b}_{l}(t)=\sum_{s} R_{l s} \frac{\delta \dot{w}_{s}\left(t-\tau_{l s}^{f *}\right)}{\tau_{s}^{*}}-\sum_{s} R_{l s} \frac{w_{s}^{*}}{\tau_{s}^{* 2}} R_{s}^{T} D_{1} \delta \dot{b}\left(t-\tau_{l s}^{f *}\right)$

which leads to

$$
\begin{aligned}
\delta \dot{w}\left(t-\tau_{l s}^{f *}\right)= & D_{2}^{-1} R_{l}^{-1} \\
& \times\left[\delta \ddot{b}_{l}(t)+\sum_{s} R_{l s} \frac{w_{s}^{*}}{\tau_{s}^{* 2}} R_{s}^{T} D_{1} \delta \dot{b}\left(t-\tau_{l s}^{f *}\right)\right] .
\end{aligned}
$$

From the last equation, the linearized system of (35) can be rewritten as

$$
\begin{aligned}
\delta \ddot{b}_{l}(t)= & -R_{l} \operatorname{diag}\left(\frac{2 w_{s}^{*}}{\tau_{s}^{*}\left(2+w_{s}^{* 2}\right)}\right) R_{l}^{-1} \\
& \times\left[\delta \ddot{b}_{l}(t)+\sum_{s} R_{l s} \frac{w_{s}^{*}}{\tau_{s}^{* 2}} R_{s}^{T} D_{1} \delta \dot{b}\left(t-\tau_{l s}^{f *}\right)\right] \\
& -\sum_{s} R_{l s} \frac{w_{s}^{*}}{\tau_{s}^{* 2}} R_{s}^{T} D_{1} \delta \ddot{b}\left(t-\tau_{l s}^{f *}\right) \\
& -\sum_{s} R_{l s} \frac{2+w_{s}^{* 2}}{2 \tau_{s}^{* 2}} R_{s}^{T} \delta \dot{p}\left(t-\tau_{l s}^{f *}-\tau_{s}^{b *}\right) .
\end{aligned}
$$

From (43), we can convert the linearized model of the AIMD model for general networks to

$$
\begin{aligned}
\delta \ddot{b}(t)=\operatorname{diag}\left(A_{2 l}\right) \dot{\delta}(t)+\sum_{s} \sum_{l}\left[\alpha_{l s} \delta \dot{b}\left(t-\tau_{l s}^{f *}\right)\right. \\
\left.+\beta_{l s} \delta \ddot{b}\left(t-\tau_{l s}^{f *}\right)+\gamma_{l s} \delta \dot{p}\left(t-\tau_{l s}^{f *}-\tau_{s}^{b *}\right)\right]
\end{aligned}
$$

that can be rewritten as the state-space model (36).

\section{ACKNOWLEDGMENT}

The author would like to thank the California Institute of Technology and the Institut National de Recherche en Informatique et en Automatique-Ecole Normale Superieure (INRIA-ENS) for their support, as this paper was written mainly at INRIA-ENS. The author would also like to thank the Associate Editor and reviewers for their helpful comments.

\section{REFERENCES}

[1] K. B. Kim and S. H. Low, Analysis and Design of AQM for Stabilizing TCP Caltech Tech. Rep. Caltech CSTR:2002.009, March 2002 [Online]. Available: http://caltechcstr.library.caltech.edu/ [Online]. Available: http://netlab.caltech.edu

[2] — , "Design of receding horizon AQM in stabilizing TCP with multiple links and heterogeneous delays," in Proc. 4th Asian Control Conf., Sep. 2002, vol. WA-1.

[3] — " "Analysis and design of AQM based on state-space models for stabilizing TCP," in Proc. IEEE Amer. Control Conf., Jun. 2003, pp. 260-265.

[4] K. B. Kim, A. Tang, and S. H. Low, "Design of AQM in supporting TCP based on the well-known AIMD model," in Proc. IEEE Globecom, Dec. 2003, vol. NG21-2.

[5] — " "A stabilizing AQM based on virtual queue dynamics in supporting TCP with arbitrary delays," in Proc. IEEE Conf. Decision and Control, Dec. 2003, vol. ThM03-2.

[6] V. Jacobson, "Congestion avoidance and control," in Proc. ACM/SIGCOMM, Aug. 1988.

[7] F. Baccelli and D. Hong, "AIMD, fairness and fractal scaling of TCP traffic," in Proc. IEEE Infocom, Apr. 2002.

[8] F. Baccelli and K. B. Kim, "TCP throughput analysis under transmission error and congestion losses," in Proc. IEEE Infocom, Mar. 2004.

[9] F. Baccelli, K. B. Kim, and D. D. Vleeschauwer, "Analysis of the competition between wired, DSL and wireless users in an access network," in Proc. IEEE Infocom, Mar. 2005.

[10] S. Floyd and V. Jacobson, "Random early detection gateways for congestion avoidance," IEEE/ACM Trans. Netw., vol. 1, no. 4, pp. 397-413, Jul. 1993.

[11] S. Floyd, "TCP and explicit congestion notification," ACM Comput. Commun. Rev., vol. 24, no. 5, pp. 10-23, 1994.

[12] W. Feng, D. Kandlur, D. Saha, and K. G. Shin, "A self-configuring RED gateway," in Proc. IEEE Infocom, Mar. 1999.

[13] T. J. Ott, T. V. Lakshman, and L. Wong, "SRED: stabilized RED," in Proc. IEEE Infocom, Mar. 1999. 
[14] M. May, T. Bonald, and J.-C. Bolot, "Analytic evaluation of RED performance," in Proc. IEEE Infocom, Mar. 2000.

[15] S. Kunniyur and R. Srikant, "End-to-end congestion control schemes: utility functions, random losses and ECN marks," in Proc. IEEE Infocom, Mar. 2000.

[16] S. Athuraliya, V. H. Li, S. H. Low, and Q. Yin, "REM: active queue management," IEEE Netw., vol. 15, no. 3, pp. 48-53, Mar. 2001.

[17] V. Misra, W. B. Gong, and D. Towsley, "Fluid-based analysis of a network of AQM routers supporting TCP flows with an application to RED," in Proc. ACM/SIGCOMM, Sep. 2000.

[18] C. Hollot, V. Misra, D. Towsley, and W.-B. Gong, "A control theoretic analysis of RED," in Proc. IEEE Infocom, Apr. 2001.

[19] _ - "On designing improved controllers for AQM routers supporting TCP flows," in Proc. IEEE Infocom, Apr. 2001

[20] S. Kunniyur and R. Srikant, "Analysis and design of an adaptive virtual queue (AVQ) algorithm for active queue management," in Proc. ACM/ SIGCOMM, Aug. 2001.

[21] H. Lim, K. J. Park, E. C. Park, and C. H. Choi, "Active queue management algorithm with a rate regulator," in Proc. 15th IFAC World Congr. Automatic Control, Jul. 2002, vol. T-We-M02.

[22] G. Vinnicombe, "On the stability of networks operating TCP-like congestion control," in Proc. 15th IFAC World Congr. Automatic Control, Jul. 2002.

[23] R. Johari and D. Tan, "End-to-end congestion control for the internet: Delays and stability," IEEE/ACM Trans. Netw., vol. 9, no. 6, pp. 818-832, Nov. 2001.

[24] L. Massoulie, "Stability of distributed congestion control with heterogeneous feedback delays," IEEE Trans. Autom. Control, vol. 47, no. 6, pp. 895-902, Jun. 2002.

[25] F. P. Kelly, A. Maulloo, and D. Tan, "Rate control for communication networks: shadow prices, proportional fairness and stability," J. Oper. Res. Soc., vol. 49, no. 3, pp. 237-252, 1998.

[26] P. Park, "A delay-dependent stability criterion for systems with uncertain time-invariant delays," IEEE Trans. Autom. Control, vol. 44, no. 4, pp. 876-877, Apr. 1999.

[27] J. Doyle, K. Glover, P. Khargonekar, and B. Francis, "State-space solution to standard $h_{2}$ and $H_{\infty}$ control problems," IEEE Trans. Autom. Control, vol. 34, pp. 831-847, 1989.

[28] M. V. Kothare, V. Balakrishnan, and M. Morari, "Robust constrained model predictive control using linear matrix inequalities," Automatica, vol. 32, pp. 1361-1379, 1996.

[29] H. Chen and F. Allgower, "A quasi-infinite horizon nonlinear model predictive control scheme with guaranteed stability," Automatica, vol. 34, pp. 1205-1217, 1998.

[30] K. B. Kim, "Design of receding horizon controls for constrained timevarying systems," IEEE Trans. Autom. Control, vol. 49, no. 12, pp. 2248-2252, Dec. 2004.

[31] P. Ranjan, E. H. Abed, and R. J. La, "Nonlinear instabilities in TCPRED," in Proc. IEEE Infocom, Jun. 2002.

[32] J. Padhye, V. Firoiu, D. Towsley, and J. Kurose, "Modeling TCP throughput: a simple model and its empirical validation," in Proc. ACM/SIGCOMM, Sep. 1998.

[33] F. Baccelli and D. Hong, "TCP is max-plus linear," in Proc. ACM/SIGCOMM, Sep. 2000.

[34] H. K. Khalil, Nonlinear Systems. Upper Saddle River, NJ: PrenticeHall, 1996.

[35] E. Altman, K. Avrachenkov, A. A. Kherani, and B. J. Prabhu, "Performance analysis and stochastic stability of congestion control protocols," in Proc. IEEE Infocom, Mar. 2005.

[36] H. Balakrishnan, V. N. Padmanabhan, S. Seshan, and R. H. Katz, "A comparison of mechanisms for improving TCP performance over wireless links," IEEE/ACM Trans. Netw., vol. 5, no. 6, pp. 756-769, Nov. 1997.

[37] S. H. Low and D. E. Lapsley, "Optimization flow control, I: basic algorithm and convergence," IEEE/ACM Trans. Netw., vol. 7, no. 6, pp. 861-874, Nov. 1999.

[38] H. Yaiche, R. R. Mazumdar, and C. Rosenberg, "A game theoretic framework for bandwidth allocation and pricing in broadband networks," IEEE/ACM Trans. Netw., vol. 8, no. 5, Sep. 2000.
[39] L. Massoulie and J. Roberts, "Bandwidth sharing: Objectives and algorithms," IEEE/ACM Trans. Netw., vol. 10, no. 3, pp. 320-328, May 2002.

[40] B. Miller, K. Avrachenkov, K. Stepanyan, and G. Miller, "Flow control as stochastic optimal control problem with incomplete information," in Proc. IEEE Infocom, Mar. 2005.

[41] G. F. Franklin, J. D. Powell, and A. E. Naeini, Feedback Control of Dynamic Systems. Reading, MA: Addison-Wesley, 1994.

[42] L. S. Brakmo and L. L. Peterson, "TCP Vegas: End to end congestion avoidance on a global Internet," IEEE J. Select. Areas Commun., vol. 13, no. 8, Aug. 1995.

[43] S. H. Low, F. Paganini, J. Wang, S. A. Adlakha, and J. C. Doyle, "Dynamics of TCP/AQM and a scalable control," in Proc. IEEE Infocom, Jun. 2002.

[44] D. H. Choe and S. H. Low, "Stabilized Vegas," in Proc. 39th Annu. Allerton Conf. Communication, Control, and Computing, Oct. 2002.

[45] F. Paganini, Z. Wang, S. H. Low, and J. C. Doyle, "A new TCP/AQM for stability and performance in fast networks," in Proc. 39th Annu. Allerton Conf. Communication, Control, and Computing, Oct. 2002.

[46] K. K. Ramakrishnan and S. Floyd, A proposal to add explicit congestion notification (ECN) to IP RFC 2481, Jan. 1999.

[47] K. B. Kim, T. W. Yoon, and W. H. Kwon, "On stabilizing receding horizon $H_{\infty}$ controls for linear continuous time-varying systems," IEEE Trans. Autom. Control, vol. 46, no. 8, pp. 1273-1279, Aug. 2001.

[48] K. B. Kim and W. H. Kwon, "Intervalwise receding horizon $H_{\infty}$ tracking controls for continuous linear time-varying systems," Proc. Inst. Elect. Eng. Control Theory Appl., vol. 151, no. 5, pp. 527-531, 2004.

[49] V. Kulkarni, M. Jun, and P. Falb, "Active queue management for TCPgoverned wireless networks," in Proc. Eur. Control Conf., Sep. 2003.

[50] J. K. Park and C. H. Choi, "Dynamic compensation method for multivariable control systems with saturating actuators," IEEE Trans. Autom. Control, vol. 40, no. 9, pp. 1635-1640, Sep. 1995.

[51] A. R. Teel, "Anti-windup for exponentially unstable linear systems," Int. J. Robust Nonlinear Control, no. 9, pp. 701-716, 1999.

[52] S. Floyd, Highspeed TCP for large congestion windows Internet draft, draft-floyd-tcp-highspeed-01.txt, 2002.

[53] T. Kelly, Scalable TCP: Improving performance in highspeed wide area networks Submitted for publication, Dec. 2002.

[54] L. Xu, K. Harfoush, and I. Rhee, "Binary increase congestion control for fast long-distance networks," in Proc. IEEE Infocom, Mar. 2004.

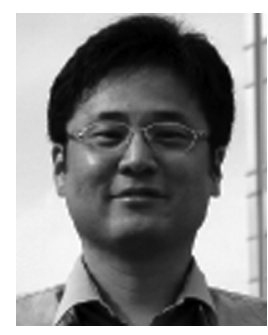

Ki Baek Kim received the B.S., M.S, and Ph.D. degrees in electrical engineering from Seoul National University (SNU), Seoul, Korea, in 1993, 1995, and 1999, respectively.

He is a Senior Engineer at the Telecommunication Network, Samsung Electronics Co., Ltd., Suwon, Korea, since September 2005. He has published several journal and conference papers in control area during his Ph.D. studies at SNU and his stay at the California Institute of Technology (CIT), Pasadena, in 1999, and at Korea University, in 2000 and 2001. In October 2001, he was with the networking area at CIT. His main research at CIT was to design active queue management based on control theory. Since February 2002, he has been with the Institut National de Recherche en Informatique et en Automatique-Ecole Normale Superieure (INRIA-ENS), Paris, France (Director F. Baccelli). His main research at INRIA-ENS has been network performance analysis based on stochastic theory, especially point process, which was a joint work with Alcatel. He is currently working on traffic control and performance analysis for WiBro (wireless broadband) systems that provide the integrated multimedia application services for wireless mobile users. 